\title{
Direct Numerical Simulations of a Smoke Cloud-Top Mixing Layer as a Model for Stratocumuli
}

\author{
Alberto de Lozar and Juan Pedro Mellado \\ Max Plank Institute for Meteorology, Hamburg, Germany
}

(Manuscript received 6 December 2012, in final form 8 April 2013)

\begin{abstract}
A radiatively driven cloud-top mixing layer is investigated using direct numerical simulations. This configuration mimics the mixing process across the inversion that bounds the stratocumulus-topped boundary layer. The main focus of this paper is on small-scale turbulence. The finest resolution $(7.4 \mathrm{~cm})$ is about two orders of magnitude finer than that in cloud large-eddy simulations (LES). A one-dimensional horizontally averaged model is employed for the radiation. The results show that the definition of the inversion point with the mean buoyancy of $\langle b\rangle\left(z_{i}\right)=0$ leads to convective turbulent scalings in the cloud bulk consistent with the Deardorff theory. Three mechanisms contribute to the entrainment by cooling the inversion layer: a molecular flux, a turbulent flux, and the direct radiative cooling by the smoke inside the inversion layer. In the simulations the molecular flux is negligible, but the direct cooling reaches values comparable to the turbulent flux as the inversion layer thickens. The results suggest that the direct cooling might be overestimated in lessresolved models like LES, resulting in an excessive entrainment. The scaled turbulent flux is independent of the stratification for the range of Richardson numbers studied here. As suggested by earlier studies, the turbulent entrainment only occurs at the small scales and eddies larger than approximately four optical lengths $(60 \mathrm{~m}$ in a typical stratocumulus cloud) perform little or no entrainment. Based on those results, a parameterization is proposed that accounts for a large part $(50 \%-100 \%)$ of the entrainment velocities measured in the Second Dynamics and Chemistry of the Marine Stratocumulus (DYCOMS II) campaign.
\end{abstract}

\section{Introduction}

The marine planetary boundary layer topped by stratocumulus clouds (STBL) is key for the planetary radiation balance (Stevens 2005; Wood 2012) and may also be important for climate change (Hartmann and Short 1980; Bony et al. 2004). In its simplest configuration the STBL consists of a lower moist boundary layer above which is a layer of much drier and warmer air: the free troposphere. The top of the STBL is populated by stratocumulus clouds that emit longwave radiation, cooling the moist boundary layer. This continuous cooling strengthens and thins the temperature inversion that separates the moist boundary layer from the free atmosphere. Radiative cooling is thought to be the main source of turbulent energy for the STBL although other processes, like evaporative cooling or drizzle, might

Corresponding author address: Alberto de Lozar, Max Plank Institute for Meteorology, Bundestr. 53, 20146 Hamburg, Germany.

E-mail: adelozar@googlemail.com substantially contribute to the STBL dynamics (Petterssen 1938; Siems and Bretherton 1992; Yamaguchi and Randall 2012).

Despite its apparent simplicity, there is still a great degree of uncertainty in current models of the stratocumulustopped boundary layer. One of the main problems arises when modeling the exchange of heat and moisture between the free atmosphere and the moist boundary layer-what has come to be known as the entrainment problem. Low-order models and climate models rely on accurate predictions of the entrainment because it determines how much energy and water is available for the STBL. Besides, the entrained air can desiccate the cloud layer in certain circumstances with important consequences for the STBL dynamics. Currently, the most popular solution is to set the entrainment efficiency (the rate of buoyancy entrained from the free atmosphere relative to a reference buoyancy flux) to a constant value: approximately 0.2 . This hypothesis is widely used in models although it has not been sufficiently validated with observations or with well-resolved models (Stevens 2002; Fedorovich et al. 2004; Caldwell et al. 2005). 
Given the difficulty of direct observations, particularly in their ability to explore the parameter space (Faloona et al. 2005), most attempts to solve the entrainment problem rely on large-eddy simulations (LES) (Lewellen and Lewellen 1998; Stevens et al. 2005; Bretherton et al. 2007; Ackerman et al. 2009; Kurowski et al. 2009; Yamaguchi and Randall 2012). LES resolve the most energetic eddies and rely on a subgrid-scale parameterization for the smaller scales, under the assumption that most of the transport is done by the largest eddies. However, Moeng et al. (1996), Stevens et al. (1999), and Stevens et al. (2005) showed that this might be not necessarily the case at the STBL inversion. They demonstrated that the entrainment fluxes strongly depend on the numerics and subgrid-scale parameterization and that the high-resolution simulations were the ones with results closest to the observations. This problem is still unresolved in contemporary LES. Heus et al. (2010) show variations of $100 \%$ in the entrainment mixing rates just by changing the advection scheme in one case study. Other cases of Heus et al. (2010) and Ackerman et al. (2009) also show a similar, although weaker, dependency on the numerics. Altogether, these results suggest that a good representation of the flow at the small scales might be helpful in understanding the cloud-top dynamics.

One of the main difficulties to model the STBL is the large number of physical processes working simultaneously. Lilly (1968) introduced a simpler, more idealized, system that captures some key dynamics of the STBL: the smoke cloud. As in stratocumulus clouds, the main driving force in the smoke cloud is the radiative cooling. The main simplification in the smoke cloud is that the evaporative cooling is neglected. An advantage of this simplified configuration is that it can be reproduced in experiments, although the typical Prandtl and Reynolds numbers in the laboratory differ largely from the atmospheric ones (Sayler and Breidenthal 1998; McEwan and Paltridge 1976). Motivated by those experiments, Bretherton et al. (1999) used smoke-cloud LES to assess the role of entrainment in the STBL. They came to the same conclusion as Stevens et al. (1999) — namely, that the unresolved small scales in the inversion are crucial for the entrainment. Contemporary smoke LES show the same problem even when the resolution was increased to $5 \mathrm{~m}$ in the horizontal and $1 \mathrm{~m}$ in the vertical (M. Khairoutdinov 2013, personal communication).

In this paper we aim to improve our physical understanding of the interaction between the radiative cooling and the inversion dynamics, focusing on the small and middle scales. With this purpose we perform direct numerical simulations (DNS) of a radiatively driven smoke cloud-top mixing layer. In this idealized setup the interaction between the radiative cooling and the inversion is isolated, while other effects (like shear, evaporative cooling, cloud base fluxes, or any action of the earth surface) are neglected. Our setup is based on the cloud-top observations made by the flight RF01 in the Second Dynamics and Chemistry of the Marine Stratocumulus (DYCOMS II) campaign (Stevens et al. 2003a,b, 2005). The main advantage of DNS is that it does not rely on any subgrid parameterization and therefore it provides a faithful representation of the turbulent small scales (Moin and Mahesh 1998). It also provides a very high resolution at the inversion: the smallest resolved scale in this paper (Kolmogorov length $\eta=7.4 \mathrm{~cm})$ is 50 times smaller than highly resolved LES (Yamaguchi and Randall 2012). The main disadvantage of DNS is that the typical Reynolds numbers that can be achieved still differ by several orders of magnitude from the atmospheric ones. This discrepancy limits DNS studies to simplified and relatively small configurations and introduces some uncertainty associated with possible low-Reynolds number effects. One of the findings presented here is that many important flow statistics, such as the turbulent and molecular buoyancy flux or the convective scalings in the cloud bulk, become indeed independent of the Reynolds number for the Reynolds numbers that we achieve. Therefore those results can be extrapolated to atmospheric values.

This paper is structured as follows. In section 2 we describe the radiatively driven smoke cloud-top mixing layer and identify the main mechanisms that trigger the flow dynamics. Section 3 presents the formulation of the problem with special emphasis on the one-dimensional radiation scheme. In section 4 we describe the general properties of the flow and define a reference position which we identify as the inversion point. This position allows us to study the inversion layer and the cloud bulk independently. Section 5 is dedicated to the study of the balance of buoyancy between the cloud bulk and the inversion layer. The inversion-layer cooling resulting from this balance is split into three components that are studied independently: the molecular flux, the turbulent flux, and the direct cooling. Section 6 extends our results to the STBL. We provide a new parameterization for the entrainment rates and compare the results to previous LES and to atmospheric measurements. In section 7 we summarize the main conclusions of the paper.

\section{Problem description}

The cloud-top mixing layer consists of a region of moist, cold air that lies below a region of dry, warm air (Fig. 1). Those regions represent the cloud and the free 


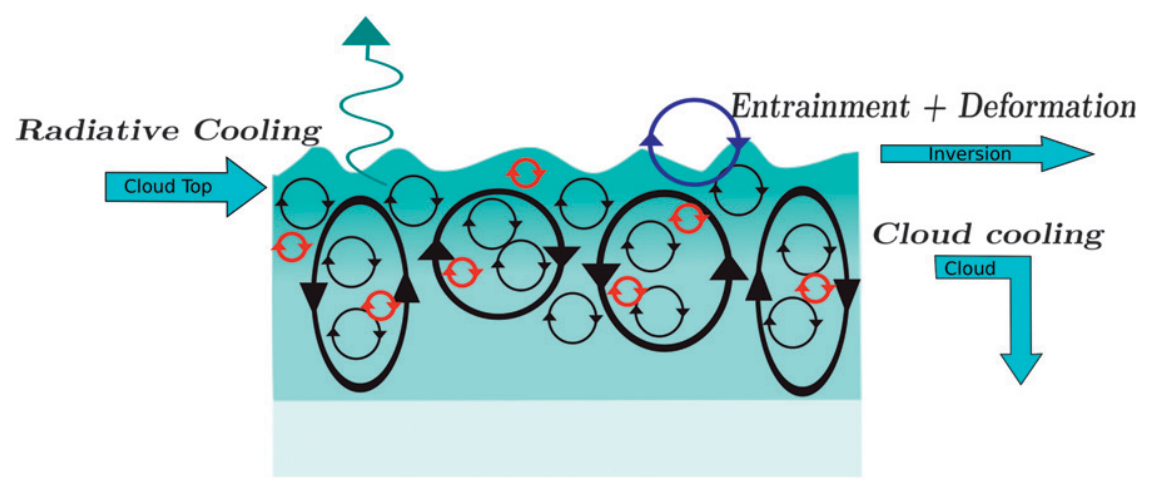

FIG. 1. Sketch of the problem. The arrows indicate the direction of the cooling. The top of the cloud emits longwave radiation at a known rate per unit surface $F_{0}$. The resulting cooled air acts at the inversion and in the cloud in different manners. At the inversion it deforms the cloud interface and cools air entrained from the free atmosphere. The rest of the cooled air falls into the cloud generating a turbulent motion while cooling the cloud. Since the turbulent motion induces the entrainment and the deformation, there is a strong interaction between the cloudbulk turbulence and the inversion dynamics.

atmosphere, respectively. Such a model configuration has already been used to investigate the evaporatively driven case (Mellado et al. 2010). We introduce two main changes with respect to the previous configuration: we use longwave radiation as the driving force for the mixing and neglect latent heat effects. With those modifications the mixing layer mimics the top of a smoke cloud.

Longwave radiation cools the air at the top of the smoke cloud over a distance approximately equal to the optical depth $\lambda$. We assume that the optical depth is much shorter than the cloud depth and that the radiation is only longwave without scattering. Under these assumptions the smoke cloud behaves as a radiative blackbody, meaning that the total emitted radiation only depends on the mean cloud temperature and on the temperature and composition of the free atmosphere. Since the free atmosphere and mean cloud temperature change slowly compared to the characteristic times associated with the cloud dynamics, the total cooling per unit surface $F_{0}$ is mostly independent of the flow in the cloud. Hence, we presume that $F_{0}$ is known and constant.

We focus on how the radiative cooling triggers the cloud dynamics and the turbulent mixing with the free atmosphere, as represented in Fig. 1. Radiatively cooled air at the cloud top begins to fall, generating a convective boundary layer (CBL) below the inversion. This CBL features eddies whose size ranges from the CBL depth down to the Kolmogorov scale. Some of those eddies interact with the inversion, entraining warm air from the free atmosphere into the cloud and deforming the interface. The CBL grows continuously in time and so does the size of the largest eddies. We focus on the initial time during which the size of the CBL is still shorter than the cloud depth. The effects of the earth surface and cloud base on the flow are thus neglected. Studying the evolution of the CBL allows us to investigate the effect of changing the integral turbulent length scale (identified as the size of the largest eddies) on the inversion dynamics. Last, depending on the stratification of the free atmosphere, gravity waves might be generated additionally but we neglect them here for simplicity.

The difficulty of the problem lies in that entrainment warms the cloud and reduces turbulence so that all processes at the cloud top are coupled. Understanding this coupling is the aim of this paper.

\section{Formulation}

\section{a. One-dimensional horizontally averaged radiation model}

We assume that the smoke that constitutes the cloud is a continuous medium characterized by an absorption coefficient $\beta_{a}$ and no scattering. We also assume that the cloud is thick enough so that the radiative exchange at the cloud bottom does not affect the cloud top. We use two main simplifications for the radiation calculations: 1) constant cloud temperature and 2) translational symmetry in the plane parallel to the cloud interface $(x-y$ plane). These approximations allow us to reduce the radiation calculations to a one-dimensional equation (e.g., Petty 2006) in which only horizontally averaged values appear. Integrating the radiative upward flux minus the downward flux in this approximation we obtain

$$
Q(z)=F_{0}\left\langle\beta_{a}\right\rangle \exp \left(-\int_{z}^{z_{\text {top }}}\left\langle\beta_{a}\right\rangle d z\right),
$$


where $Q(z)$ is the radiative cooling per unit volume at height $z, F_{0}$ is the energy exchange of the cloud with the atmosphere (per unit surface), $z_{\text {top }}$ is the cloud top, and the angle brackets symbolize the horizontal average. Because of their simplicity, similar expressions are commonly used in LES codes to deal with the longwave radiation (Larson et al. 2007; Heus et al. 2010) and the results obtained are consistent with observations. For a suspension of spherical particles whose radius $r$ is larger than $5 \mu \mathrm{m}$, the absorption coefficient has a simple expression of the form

$$
\beta_{a}=\frac{3}{2 \rho_{s} r} q_{s} \rho_{t}=\kappa q_{s} \rho_{t},
$$

where $\rho_{s}$ is the density of the smoke, $\rho_{t}$ is the density of the cloudy air, $q_{s}$ is the mass fraction of smoke, and $\kappa$ is a constant. The constant $\kappa$ is introduced because some radiation models use Eq. (1) but with a more complex expression for $\kappa$ than the one given by Eq. (2) (Larson et al. 2007).

Let us briefly discuss the main approximations used in the radiation model. We assume that the constantcloud-temperature assumption does not significantly modify the final results. As the Stephan-Boltzmann law indicates, the radiation emitted by a cloud parcel goes as $Q \sim T^{4}$. For typical cloud-top temperature variations $(\delta T \sim 1 \mathrm{~K})$, the cooling function deviates only by a small percentage $(\delta Q / Q \sim 1 \%)$ from the mean value. Besides, we expect that these deviations will quickly be smoothed owing to the turbulence inside the cloud. The error introduced by the translational symmetry at the cloud-top is more difficult to quantify. If the cloud interface is strongly convoluted, the radiation model artificially cools parcels of dry air that are close to the cloud while at the same time artificially reduces the cooling of the cloud parcels close to the interface. However, simple models like this one can still produce reliable results, even when the instantaneous cooling function significantly deviates from the correct one. Pincus and Stevens (2009) show that turbulence smooths out the cooling function deviations and that simple radiation models tend to match the averaged cooling profiles obtained in more complex models. Further work will address this issue and investigate the importance of the radiation formulation for the cloud-top dynamics. In this paper we only use the averaged formulation.

Assuming a constant cloud density $\rho_{t}$, Eqs. (1) and (2) are rewritten introducing a reference length $\lambda$ :

$$
\begin{aligned}
& Q(z)=\frac{F_{0}}{\lambda}\langle f(z)\rangle e^{-\tau(z)}, \\
& \tau(z)=\frac{1}{\lambda} \int_{z}^{z_{\text {top }}}\left\langle f\left(z^{\prime}\right)\right\rangle d z^{\prime},
\end{aligned}
$$

$$
\lambda=\frac{1}{\kappa \rho_{t} q_{s}^{c}},
$$

where $f=q_{s} / q_{s}^{c}$ is the normalized proportion of smoke, $q_{s}^{c}$ is the smoke concentration in the cloud bulk, and $\tau(z)$ is the vertical optical path between $z$ and the top of the cloud. The optical depth, also called optical length, is equal to the distance that incident radiation travels through a cloud of constant smoke density before decaying by a factor 1/e. As a result, the top layer of the cloud, which is directly cooled by radiation, is on the order of this distance. Notice that the volumetric integral of Eq. (3) for the whole domain yields $\iint Q d z d S=F_{0} S$, where $S$ is the horizontal surface of the domain. This means that the total cooling of the cloud per unit of surface is constant and equal to $F_{0}$, as expected from a blackbody.

\section{b. Evolution equations}

We use the evolution equations in the Boussinesq approximation because of the small density variations over the domains considered in this work. The equation for the buoyancy can be derived from the enthalpy conservation equation in the Boussinesq approximation, under a two-continuum formulation, with the addition of the radiation source term given by Eq. (3). The equation for the smoke is just an advection-diffusion equation. The resulting system of equations reads

$$
\begin{aligned}
\frac{\partial \mathbf{v}}{\partial t}+(\mathbf{v} \cdot \nabla) \mathbf{v} & =-\nabla p+\nu \nabla^{2} \mathbf{v}+b \mathbf{k} \\
\nabla \cdot \mathbf{v} & =0 \\
\frac{\partial b}{\partial t}+(\mathbf{v} \cdot \nabla) b & =\kappa_{t} \nabla^{2} b-\frac{B_{0}}{\lambda}\langle f\rangle e^{-\tau(z)} \\
\frac{\partial f}{\partial t}+(\mathbf{v} \cdot \nabla) f & =\kappa_{s} \nabla^{2} f
\end{aligned}
$$

where $\mathbf{v}$ is the velocity field, $p$ is a modified pressure divided by a reference cloud density $\rho_{c}, b$ is the buoyancy $b=g\left(T-T_{c}\right) / T_{c}, T_{c}$ is the reference cloud temperature, $\mathbf{k}$ is the unity vector in the vertical direction, $\nu$ is the kinematic viscosity, $\kappa_{t}$ is the thermal diffusivity, $\kappa_{s}$ is the smoke diffusivity, and $B_{0}$ is the reference buoyancy flux given by radiation:

$$
B_{0}=\frac{F_{0} g}{\rho_{c} c_{p} T_{c}},
$$

where $g$ is the acceleration of gravity and $c_{p}$ is the constant pressure heat capacity of cloudy air. 


\section{c. Boundary conditions and initial conditions}

In the lower layer, the cloud bulk, the smoke concentration is equal to the cloud value $\left[f\left(z_{\text {bot }}\right)=1\right]$ and the temperature is the cloud temperature resulting in a zero buoyancy $\left[b\left(z_{\text {bot }}\right)=0\right]$. In the upper layer, the free atmosphere, there is no smoke $\left[f\left(z_{\text {top }}\right)=0\right]$ and the temperature is higher than in the cloud resulting in a positive buoyancy $\Delta b$.

Stretched grids were used to put the boundaries far from the cloud interface in order to avoid any influence of the boundaries or the boundary conditions on the cloud dynamics. We use no penetration, free-slip boundary conditions for the velocity. The boundary conditions for the scalars are Dirichlet for the smoke (constant smoke) and Neumann for the buoyancy (constant flux).

For the initial condition we connect the two layers using a smooth transition. We use a hyperbolic tangent profile centered around $z_{0}$ over a thickness $\delta$. The temperature field is shifted with respect to the scalar field by a distance $\theta$, as observed in our results (shown below). The mathematical expression of this initial condition reads

$$
\begin{gathered}
f_{\text {i.c. }}(z)=\left[1-\tanh \left(\frac{z-z_{0}}{\delta}\right)\right] / 2, \\
b_{\text {i.c. }}(z)=\Delta b\left[\tanh \left(\frac{z-z_{0}+\theta}{\delta}\right)+1\right] / 2 .
\end{gathered}
$$

The initial conditions were chosen with the objective of reaching the self-preserving state, which is independent of the initial condition, as soon as possible. Consequently, we fixed $\delta=0.1 \lambda$ and $\theta=0.05 \lambda$, as suggested by additional simulations in smaller configurations (not shown here). The buoyancy profile was modified to include the effect of the radiative cooling over two time units (see below) without any mixing. We add a velocity perturbation characterized by a Gaussian power spectral density centered at a spatial frequency equal to the reciprocal of the optical depth $1 / \lambda$. The resulting configuration mimics a three-layer system whose middle layer, of thickness similar to $\lambda$, is buoyantly unstable. The velocity perturbation triggers the unstable mode of frequency $1 / \lambda$ as shown by the instability analysis in Mellado et al. (2009).

\section{d. Implementation}

The transport equations, written in Cartesian coordinates, are solved using finite differences on a structured mesh that is isotropic in the central part of the domain where the flow is turbulent. The numerical algorithm is based on a low-storage fourth-order Runge-Kutta scheme (Carpenter and Kennedy 1994) and sixth-order, spectral-like compact finite differences (Lele 1992). At the boundaries of the vertical nonperiodic direction, they are biased with third-order accurate formulas, leading to global fourth-order accuracy in space (Carpenter et al. 1993). The pressure-Poisson equation is solved using a Fourier decomposition along the periodic horizontal planes and a factorization of the resulting set of equations along the vertical coordinate (Mellado and Ansorge 2012). This choice is motivated by the high resolving efficiency and minimal numerical diffusion of the compact schemes, which makes them computationally more attractive than low-order schemes despite their implicit character, in particular, for the specific study of the cloud top (Dietze et al. 2012). For instance, the level of $1 \%$ discretization error in the phase velocity is achieved with six points per wavelength in the compact schemes here used, whereas a centered second-order scheme requires more than 20 points.

All of the simulations discussed in this paper have a resolution parameter $\Delta x / \eta$ on the order of 2.0 or less, where $\Delta x$ is the grid spacing and $\eta$ is the Kolmogorov length. Using grid convergence studies (not shown), such a resolution has been proved to be enough for accuracies on the order of $2 \%$ or better in the statistics discussed in this paper, using the numerical algorithm described above. Further details can be found in Mellado (2010), where a thorough discussion of such a validation procedure is included in the appendix.

\section{e. Adimensional groups}

The radiation formulation introduces a reference length scale $\lambda$ and a reference buoyancy flux $B_{0}$ into the problem. Based on these quantities we can construct a velocity scale, a time scale, and a buoyancy scale:

$U_{0}=\left(B_{0} \lambda\right)^{1 / 3}, \quad t_{0}=\left(\lambda^{2} / B_{0}\right)^{1 / 3}, \quad b_{0}=\left(B_{0}^{2} / \lambda\right)^{1 / 3}$.

These reference scales correspond to the velocity and turnover time of eddies whose size is equal to the optical length, under the assumption that the energy cascade follows the Kolmogorov inertial scaling (Pope 2000) and that the dissipation rate is equal to $B_{0}$. These scales therefore describe the eddies that are thought to interact the most with radiation. Based on these scales we produce four nondimensional numbers, which define the problem uniquely once the flow becomes independent of the initial conditions:

$$
\begin{aligned}
\mathrm{Ri}_{0} & =\lambda \Delta b / U_{0}^{2}=\Delta b / b_{0}, \\
\operatorname{Re}_{0} & =U_{0} \lambda / \nu, \\
\operatorname{Pr} & =\nu / \kappa_{t}, \\
\mathrm{Sc} & =\nu / \kappa_{s},
\end{aligned}
$$


TABLE 1. Simulation details. The stratification and the viscous forces are characterized by a reference Richardson number $\mathrm{Ri}_{0}$ and by a reference Reynolds number $\mathrm{Re}_{0}$, respectively. All simulations were done for $\mathrm{Sc}=\operatorname{Pr}=1$. The third column shows the domain size in dimensions of optical depths, differentiating between the horizontal and the vertical extension. The fourth column represents the number of points of the numerical grid. The fifth column represents the duration of each simulation $\left[t_{0}\right.$ is a reference time defined in Eq. (13) and for DYCOMS II $t_{0} \sim 50 \mathrm{~s}$ ]. All next columns describe the final stage of each simulation. The sixth, seventh, and eighth columns indicate the typical size of the largest eddies $\left(z^{*}\right)$, the smallest eddy size (the Kolmogorov scale), and the temperature stratification when the problem is dimensionalized with an optical length $\lambda=15 \mathrm{~m}$. The last two columns present the final convective Reynolds and Richardson numbers (see text).

\begin{tabular}{|c|c|c|c|c|c|c|c|c|c|}
\hline $\mathrm{Ri}_{0}$ & $\mathrm{Re}_{0}$ & Domain size & Numerical grid & $t / t_{0}$ & $z^{*}(\mathrm{~m})$ & $\eta(\mathrm{cm})$ & $\Delta T(\mathrm{~K})$ & $\mathrm{Re}^{*}$ & $\mathrm{Ri}$ * \\
\hline 10 & 90 & $(90 \lambda)^{2} \times 90 \lambda$ & $1024^{2} \times 1024$ & 31.5 & 480 & 69.3 & 1.75 & 7300 & 50 \\
\hline 10 & 400 & $(30 \lambda)^{2} \times 30 \lambda$ & $1024^{2} \times 1024$ & 16.8 & 170 & 21.0 & 1.75 & 8700 & 29 \\
\hline 10 & 800 & $(36 \lambda)^{2} \times 18 \lambda$ & $2048^{2} \times 1024$ & 12.9 & 120 & 12.3 & 1.75 & 12100 & 24 \\
\hline 10 & 1600 & $(18 \lambda)^{2} \times 18 \lambda$ & $2048^{2} \times 2048$ & 11.7 & 110 & 7.4 & 1.75 & 21300 & 23 \\
\hline 57 & 400 & $(30 \lambda)^{2} \times 30 \lambda$ & $1024^{2} \times 1024$ & 17.5 & 190 & 21.8 & 10 & 9900 & 190 \\
\hline 57 & 800 & $(36 \lambda)^{2} \times 18 \lambda$ & $2048^{2} \times 1024$ & 11.1 & 120 & 13.1 & 10 & 10900 & 150 \\
\hline 57 & 1600 & $(18 \lambda)^{2} \times 18 \lambda$ & $2048^{2} \times 2048$ & 12.0 & 120 & 7.5 & 10 & 23000 & 140 \\
\hline
\end{tabular}

where $\mathrm{Ri}_{0}$ is a reference Richardson number, $\mathrm{Re}_{0}$ is a reference Reynolds number, Pr is the Prandtl number, and $\mathrm{Sc}$ is the Schmidt number. The reference Richardson number is the Richardson number associated with eddies of size $\lambda$ and provides an estimate for the strength of the stratification. The reference Reynolds number provides an estimate for the scale separation between the radiative scale and the turbulent dissipation scale $\left[\operatorname{Re}_{0} \simeq(\lambda / \eta)^{4 / 3}\right]$. In all our simulations we set the Prandtl and Schmidt numbers equal to one and therefore we refer to each case just by quoting the reference Reynolds and Richardson numbers.

We use the measurements of the DYCOMS II campaign to estimate reference values for $\mathrm{Ri}_{0}$ and for $\mathrm{Re}_{0}$. The thermodynamic state is characterized by $T_{c}=11^{\circ} \mathrm{C}$ and a liquid water content $q_{1}=0.45 \mathrm{~g} \mathrm{~kg}^{-1}$, with an inversion strength varying between $\Delta T=6^{\circ} \mathrm{C}$ and $\Delta T=$ $11^{\circ} \mathrm{C}$, as observed for different flights. The estimation of the radiation parameters depends on the radiation model used to fit the observations and certain scatter exits in the literature. Stevens et al. (2005) found that the parameters $\left(F_{0}=70 \mathrm{~W} \mathrm{~m}^{-2}, \kappa=85 \mathrm{~m}^{2} \mathrm{~kg}^{-1}\right)$ fitted best the modeled radiation flux profiles for the flight RF01 of DYCOMS II, while Larson et al. (2007) obtained $\left(F_{0}=62 \mathrm{~W} \mathrm{~m}^{-2}\right.$ and $\left.\kappa=119 \mathrm{~m}^{2} \mathrm{~kg}^{-1}\right)$ using a different radiation model. This second set of values agrees better with the set previously presented by Stephens (1978) $\left(\kappa=158 \mathrm{~m}^{2} \mathrm{~kg}^{-1}\right)$ for a more general case. This uncertainty introduces a big variation for $\lambda$ (between 10 and $25 \mathrm{~m}$ ) but not so much in $B_{0}$, whose typical value is $B_{0} \sim$ $1.9 \times 10^{-3} \mathrm{~m}^{2} \mathrm{~s}^{-3}$. Using $\lambda=15 \mathrm{~m}$ the corresponding typical reference velocities are on the order of $U_{0} \sim$ $0.3 \mathrm{~m} \mathrm{~s}^{-1}$ and reference times are on the order of $t_{0} \sim 50 \mathrm{~s}$. Typical reference Richardson numbers vary in the interval $30<\mathrm{Ri}_{0}<60$ and typical reference Reynolds number are on the order of $\mathrm{Re}_{0}=2 \times 10^{5}$.
All simulations are summarized in Table 1. We focus in this paper on two limiting stratifications: $\mathrm{Ri}_{0}=10$ and $\mathrm{Ri}_{0}=57$. The higher stratification corresponds to a temperature inversion on the high end of the observations in stratocumulus while the lower stratification was chosen to investigate how the flow statistics vary by changing the stratification. We investigate three reference Reynolds numbers $\left(\operatorname{Re}_{0}=400,800,1600\right)$ for each stratification. By increasing the reference Reynolds number we reduce the Kolmogorov scale to less than $10 \mathrm{~cm}$, as shown in Table 1. Though still much larger than atmospheric values $\left(\eta_{\text {atm }} \sim 1 \mathrm{~mm}\right)$, these scales are still much smaller than typical resolutions in highly resolved LES (around $5 \mathrm{~m}$ ). The largest scale of the flow, given by the integral flow scale $z^{*}$ defined in section 5 , is around $150 \mathrm{~m}$. To investigate the influence of the integral scale size on the inversion we also perform a simulation with a lower reference Reynolds number $\left(\mathrm{Re}_{0}=90\right)$, which allows us to reach much larger integral scales. Such a simulation is not possible with the higher stratification because viscous effects dominate at the inversion for the combination of high $\mathrm{Ri}_{0}$ and low $\mathrm{Re}_{0}$.

\section{General properties}

\section{a. Visualizations}

In Fig. 2 we show vertical cross sections of the buoyancy field for the two stratifications studied in this paper. The fluid is cooled at the cloud top and falls into the cloud bulk forming a convective boundary layer. This CBL advances downward featuring eddies of multiple sizes. The plots of the vertical velocity (not shown here) show that the updrafts and downdrafts have similar width and strength. Moeng and Schumann (1991) showed that this similitude is typical of the STBL, in 

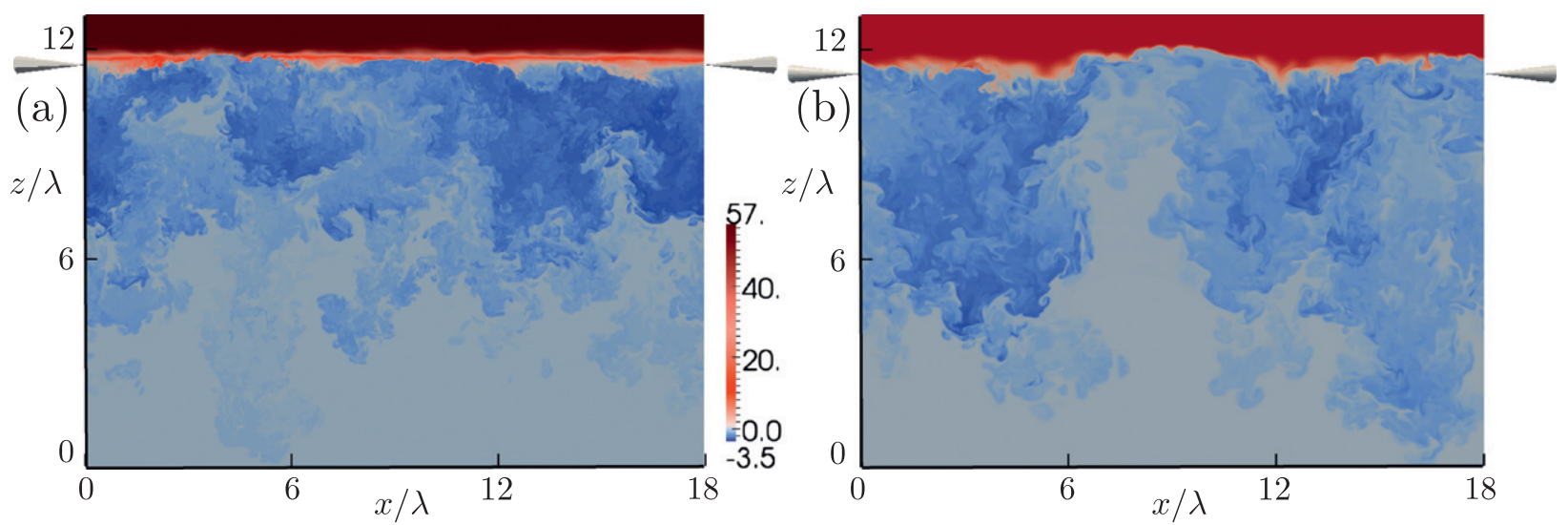

FIG. 2. Vertical cross sections of the buoyancy field for (a) a high stratification $\left(\mathrm{Ri}_{0}=57, \mathrm{Re}_{0}=1600\right)$ and $(\mathrm{b})$ a low stratification $\left(\mathrm{Ri}_{0}=\right.$ $10, \mathrm{Re}_{0}=800$ ) case at the last stage of the simulation (see Table 1). The cones indicate the position of the inversion point $z_{i}$ using the definition explained in the text. The color scale has been chosen to emphasize the flow structures: the blue scale ranges from $b=-3.5 b_{0}$ to $b=0$, the red scale from $b=0$ to $b=10 b_{0}$, and the magenta scale from $b=10 b_{0}$ to $b=57 b_{0}$.

contrast to the well-differentiated updrafts and downdrafts that characterize the dry CBL.

At the cloud top some of the eddies are able to entrain dry air from the free atmosphere, warming the cloud. The images suggest that only small eddies are capable to directly engulf dry air at the inversion layer, both in the high and in the low stratification cases. The largest eddies hit and deform the inversion (one of these events is very clear in the center of Fig. $2 b$ ) but they do not seem to engulf any air from above. The deformation is clearer in the low stratification while in the high stratification the inversion keeps a relatively flat interface at all times. Large eddies might contribute to the entrainment through secondary processes (like shear-induced instabilities) but those are difficult to identify in the flow visualizations. This flow description is common for all simulations we have done and it is quantified in the following sections.

A horizontal cross section of the smoke field is shown in Fig. 3. The smoke field presents a clear cellular pattern where wide regions of almost pure smoke $(s \sim 1)$ are separated by very thin borders with much lower smoke concentrations. According to our observations, the wavelength of the pattern is similar to the boundary layer height. This cellular pattern is common in turbulent convection (Flack et al. 2001; Chillà and Schumacher 2012) and reminiscent of stratocumulus.

\section{b. Mean profiles at the inversion layer}

One advantage of the highly resolved simulations is that they provide a more detailed picture of the inversion layer. In Fig. 4 we present the mean profiles of radiative forcing, smoke, and buoyancy together with the mean turbulent and molecular fluxes of the same scalars. For these plots we chose the case previously shown in Figs. 2 and $3\left(\mathrm{Ri}_{0}=10, \mathrm{Re}_{0}=800\right)$, but the qualitative trends explained here are common to all the simulations.

In Fig. 4 it is useful to introduce a reference height, the inversion point $z_{i}$, which separates the inversion layer from the CBL. However, there is not a unique definition for this height. The inversion point is defined in this paper as the point where the horizontally averaged profile of buoyancy is equal to zero $\left[\langle b\rangle\left(z_{i}\right)=0\right]$. This definition of the inversion is based on how cloud parcels react to the radiative cooling. Above the inversion point,

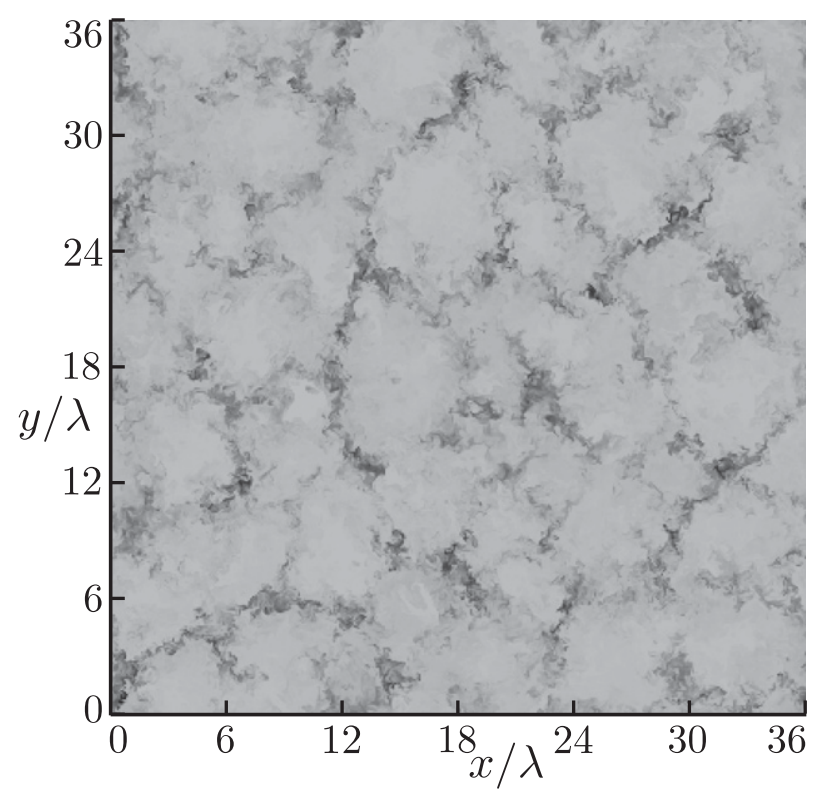

FIG. 3. Horizontal cross section of the smoke field. The color scale ranges from $s=0.4$ (black) to $s=1.0$ (white). The section was extracted from a horizontal cut one optical length below the inversion $\left(z=z_{i}-\lambda\right)$. The parameters of the simulation are $\left(\mathrm{Ri}_{0}=\right.$ $\left.10, \operatorname{Re}_{0}=800\right)$. 

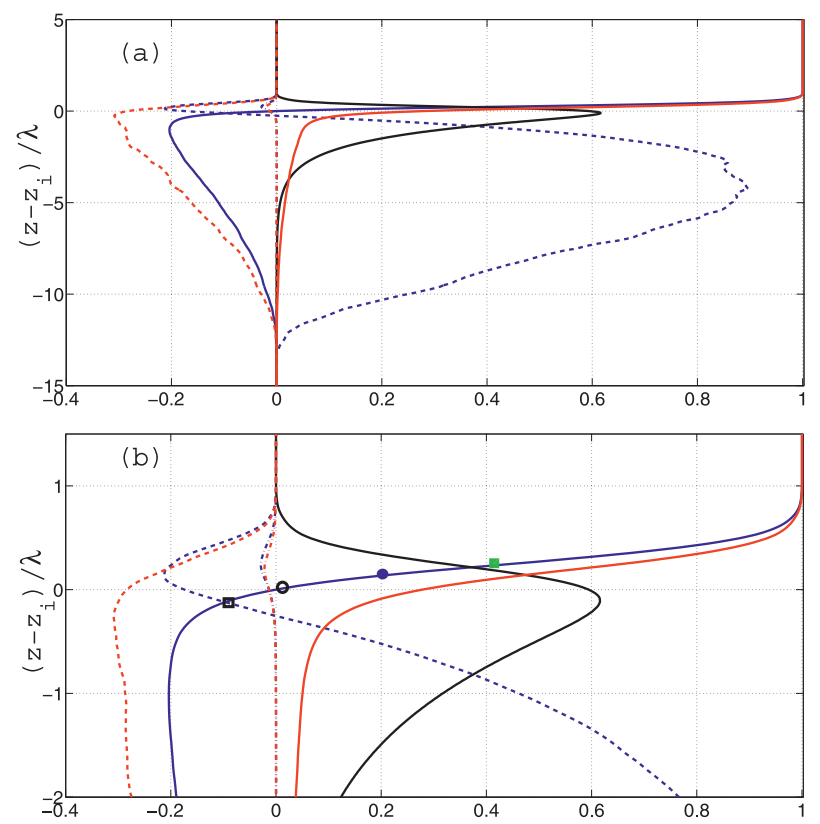

FIG. 4. (a) Horizontally averaged flux and mean profiles at the instant $t=12.95 t_{0}$ in the simulation $\left(\mathrm{Ri}_{0}=10, \mathrm{Re}_{0}=800\right)$. (b) Zoom close to the inversion. The color codes the scalar: blue for smoke and red for buoyancy. The type of line codes the property: solid for mean profiles, dashed for turbulent fluxes, and dashed-dotted for molecular fluxes. The black line shows the cooling function. The mean profile of buoyancy has been scaled by the inversion jump $\Delta b$; the mean smoke profile has been transformed to vary from 0 to $1(1-f$ is shown); the molecular and turbulent buoyancy fluxes are scaled by $B_{0}$; and the smoke fluxes are scaled by $-B_{0} / \Delta b$. The symbols represent different choices for the inversion point: maximum slope of the mean buoyancy profile (solid square), minimum turbulent flux (solid circle), zero buoyancy (open circle), and maximum of the radiative function (open square).

radiation cools parcels whose mean temperature is higher than the cloud temperature, and therefore the radiative cooling moves the inversion in the temperature profile upward, into the free atmosphere. On the other hand, parcels cooled below the inversion point are in average less buoyant than the cloud and the radiative cooling helps these parcels to fall into the cloud. The justification and implications of this choice for the inversion point are discussed throughout the paper.

The buoyancy profile in Fig. 4 presents a minimum close to the inversion point and then increases progressively until zero far down the boundary layer. The presence of this minimum was already observed by Sayler and Breidenthal (1998) in a tank experiment driven by radiation, although in the experiments the temperature drops more abruptly to the in-cloud temperature. In Fig. 4 the smoke profile is shifted by around $0.1 \lambda$ with respect to the buoyancy profile. This shift is consistent for all simulations we performed. The smoke profile enters the inversion layer so that the region that is directly cooled by radiation extends into the inversion layer. We discuss in the next sections the implications of this direct cooling of the inversion.

The negative peak of the buoyancy turbulent flux is placed close above the inversion point, and the maximum of the smoke turbulent flux is located close below the inversion point. This differs from zero-order entrainment models that assume that both peaks are located at the inversion point and have the same magnitude when using the scaling described in the caption of Fig. 4. We observe instead that the scaled maximum turbulent flux of smoke is higher in magnitude than the negative peak of the scaled turbulent buoyancy flux. This indicates that the turbulent transport into the inversion layer is more efficient for smoke than for buoyancy. We expand this point in section 5 .

At the inversion point, the molecular flux is much lower than the turbulent flux for all the simulations that we performed. In some cases (as in Fig. 4) this happens for all points along the inversion layer. This is however not the case if the Richardson number is high enough or for low Reynolds numbers. For those cases the turbulent flux drops inside the inversion layer where it is overtaken by the molecular flux (not shown here). In just those cases molecular flux is the main mechanism for transport of buoyancy from approximately the point of maximum slope of the mean buoyancy profile (at the middle of the inversion layer) to the free atmosphere, even when the transport at the inversion point is still clearly dominated by the turbulent flux. Understanding this relative importance of the Richardson and Reynolds numbers is precisely one of the goals of the present paper.

\section{The inversion energy balance}

One of the main problems in the study of the STBL inversion energy balance, which leads to discrepancies of order one, is the definition of the inversion point (Lilly 2002; Fedorovich et al. 2004; Moeng et al. 2005). In Fig. 4 we show the position of our choice for the inversion point $\left[\langle b\rangle\left(z_{i}\right)=0\right]$ together with other plausible definitions. Notice that the reference values of the fluxes and profiles at the inversion point change substantially depending on the choice of the inversion point. Each of those points defines an energy balance but not every balance is relevant for the total energy exchange as defined by Lilly (1968). The challenge is to demonstrate that our chosen inversion point provides a relevant balance equation.

Once the inversion point is defined, the total cooling of the system can be divided into the cooling of the cloud bulk and the cooling of the inversion layer: 


$$
\begin{aligned}
-B_{0} & =\frac{\partial}{\partial t} \int_{0}^{z_{i}}\langle b(z)\rangle d z+\frac{\partial}{\partial t} \int_{z_{i}}^{\infty}\langle b(z)\rangle d z \\
& =Q_{\mathrm{cbl}}(t)+Q_{\text {inv }}(t) .
\end{aligned}
$$

If this partition is relevant for our problem, the convective boundary layer that forms below the inversion should react only to the injected buoyancy flux $Q_{\mathrm{cbl}}(t)$, justifying our definition of the inversion point. This is the topic of this section.

\section{a. The convective boundary layer in the cloud bulk}

The velocity, buoyancy, and length scales in an convective boundary layer are described by the convective scales proposed by Deardorff (1970a,b). The definition of these convective scales requires the introduction of a reference buoyancy flux $B_{\text {ref }}$, which is equal to the buoyancy flux injected into the CBL. If the inversion point is well defined, the cooling rate $Q_{\mathrm{cbl}}(t)$ should be a good candidate for the convective reference flux $B_{\text {ref }}$. The only drawback of this definition is that $Q_{\mathrm{cbl}}(t)$ is an instantaneous measure. Since the CBL requires some time to adapt to the changes in the inversion layer, we expect that the CBL dynamics are more sensitive to the integrated value of $Q_{\mathrm{cbl}}(t)$, which quantifies the total energy injected into the CBL. We thus propose for the reference flux an averaged flux of the type

$$
B_{\mathrm{ref}}(t)=-\left[\int_{t_{i}}^{t} Q_{\mathrm{cbl}}\left(t^{\prime}\right) d t^{\prime}\right] /\left(t-t_{i}\right),
$$

where $t_{i}$ is the initial time of the simulations. In general the integration time $\left(t-t_{i}\right)$ is twice as long as the largeeddy turnover time $w^{*} / t^{*}$ (defined below). The integration time is therefore similar to the time that requires the CBL to adapt to variations in the injected buoyancy flux.

Once the reference flux is known, we define the convective scales as

$$
\begin{aligned}
z^{*} & =\frac{1}{B_{\mathrm{ref}}} \int\left\langle w^{\prime} b^{\prime}\right\rangle d z, \\
w^{*} & =\left(B_{\mathrm{ref}} z^{*}\right)^{1 / 3}, \\
b^{*} & =B_{\mathrm{ref}} / w^{*}
\end{aligned}
$$

where the asterisk refers to the convective scales, the primes to fluctuation values, and the integral extends over the whole domain. The length $z^{*}$ is usually identified with the size of the boundary layer and therefore it is similar to the integral length scale. The buoyancy $b^{*}$ scales the mean and rms buoyancy in the boundary layer and $w^{*}$ is associated to the velocity of the largest eddies of size $z^{*}$.
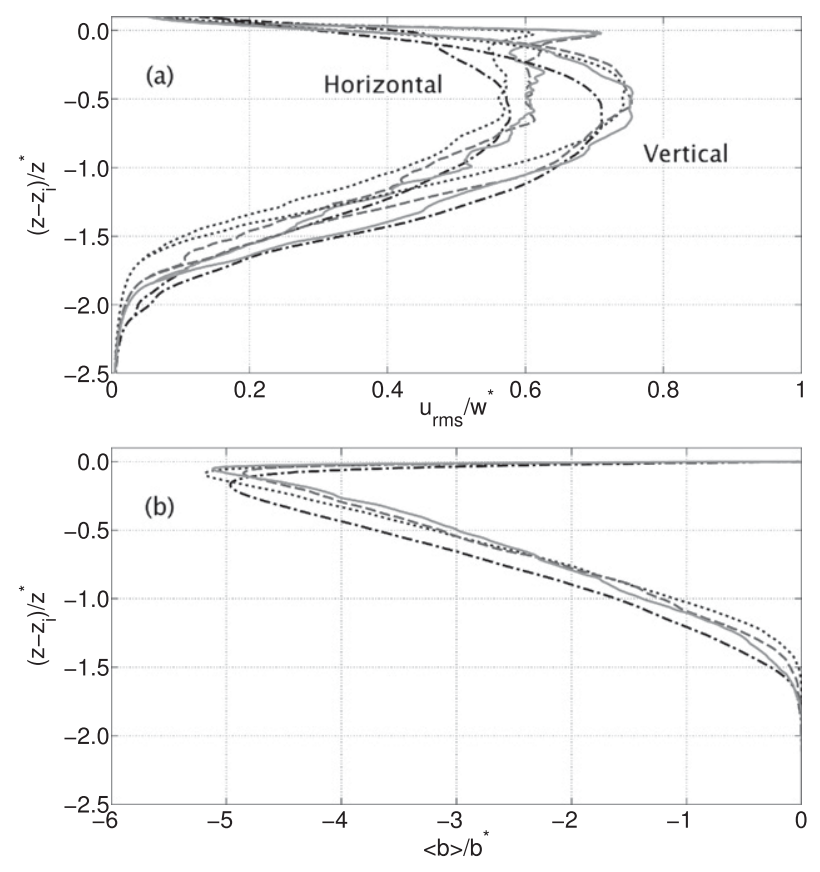

FIG. 5. Self-similar vertical profiles of the (a) velocity fluctuations and (b) buoyancy, plotted for different times in the case $\left(\mathrm{Re}_{0}=90, \mathrm{Ri}_{0}=10\right)$. The velocity fluctuations are divided into their horizontal component $u_{\mathrm{rms}}=\sqrt{\left\langle u^{\prime} u^{\prime}\right\rangle+\left\langle v^{\prime} v^{\prime}\right\rangle}$ and their vertical component $w_{\text {rms }}=\sqrt{\left\langle w^{\prime} w^{\prime}\right\rangle}$. The correspondence of the lines to the time and convective length scale is the following: dasheddotted line for $t=7.9 t_{0}, z^{*}=6.7 \lambda$; dotted line for $t=16.4 t_{0}, z^{*}=$ 15.8ג; dashed line for $t=23.0 t_{0}, z^{*}=22.1 \lambda$; and the solid line for $t=29.4 t_{0}, z^{*}=28.1 \lambda$.

In the convective regime, averaged flow properties scaled with the convective scales are independent of time and just depend on the self-similar variable $\xi=\left(z-z_{i}\right) / z^{*}$. In Fig. 5 we show the scaled velocity fluctuations and mean buoyancy as a function of $\xi$ for the case $\left(\mathrm{Ri}_{0}=10\right.$, $\mathrm{Re}_{0}=90$ ). We choose the lowest reference Reynolds number simulation to present this result because it extends the longest in time after the initial transient. Notice that the boundary layer quadruples its size after the initial transient, from $z^{*} \sim 7 \lambda$ to $z^{*} \sim 28 \lambda$, but that all scaled profiles collapse to the same curve. Buoyancy fluctuations and other second-order statistics also collapse (not shown). The scaled size of the boundary layer remains constant at $1.5-2.0 z^{*}$, which justifies the definition of $z^{*}$ as the CBL depth. Two main conclusions can be drawn from these results. First, the excellent collapse of the curves suggests that our choices for the inversion point and for $B_{\text {ref }}$ are appropriate. Second, it indicates that the convective Reynolds number reached in this simulation is high enough to attain the inviscid scaling. This allows us to extrapolate the results for higher Reynolds numbers.

Using again the convective scaling we compare the mean buoyancy and velocity fluctuation profiles for all 

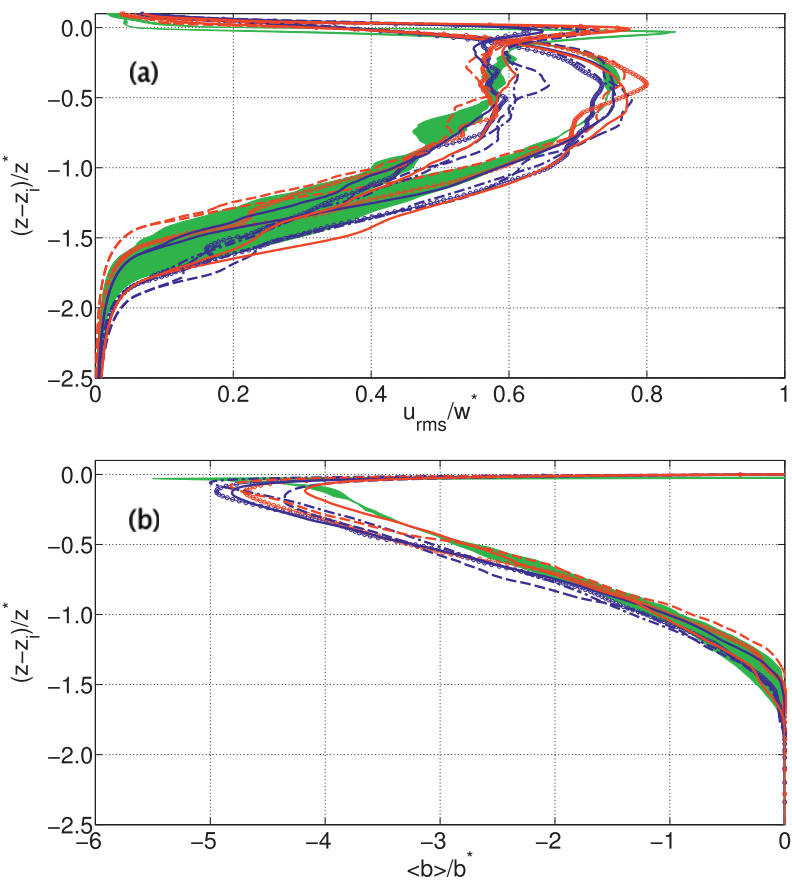

FIG. 6. Self-similar vertical profiles of the (a) velocity fluctuations and (b) buoyancy, plotted for different Reynolds and Richardson numbers. The profiles are averaged from $z^{*} \sim 6 \lambda$, once the boundary layer reaches the convective regime. The meaning of the axis is as in Fig. 5. The color codes the Richardson number: red for $\mathrm{Ri}_{0}=57$ and blue for $\mathrm{Ri}_{0}=10$. The type of line codes the Reynolds number: points over lines for $\mathrm{Re}_{0}=1600$, solid for $\mathrm{Re}_{0}=800$, dashed for $\mathrm{Re}_{0}=400$, and dashed-dotted for $\mathrm{Re}_{0}=90$. The shaded area shows the result for a boundary layer driven by evaporative cooling from Mellado (2010).

cases in Fig. 6. In each case the profiles are averaged over several time steps, starting from the time the selfsimilar regime was reached (from $z^{*}=6 \lambda$ approximately). The simulations vary greatly in stratification, Reynolds number, and initial conditions. However, all curves collapse, meaning that the flow in the boundary layer is independent of the inversion details. The only necessary parameter is the total flux into boundary layer $B_{\text {ref }}$, which appears in the convective scaling. Our choice for $B_{\text {ref }}$ seems to capture the flow dynamics in the CBL for all cases, confirming our election of the inversion point. Other choices for the inversion point (maximum gradient of buoyancy, minimum buoyancy turbulent flux, or the point at which any of the scalars reach $50 \%$ of the free-atmosphere value) do not reproduce the convective scalings as shown here. From those alternative choices, just the minimum buoyancy turbulent flux seems to reproduce the convective scaling to some extent (although the collapse of the curves is worse than shown here), probably because this point is always near to the point of zero buoyancy.
We also compare our scaled results to the simulations by Mellado (2010), where the cloud was cooled by evaporative cooling alone (shaded area in Fig. 6). The width of the shaded area represents the typical variations owing to lack of statistical convergence. For clarity we show only the variations in the results of Mellado (2010), although our simulations present similar variations. The agreement of the evaporative results with our simulations is very good. We conclude that the dynamics of the convective boundary layer decouple from the inversion so that in a first approximation the boundary layer dynamics only depends on the absolute flux of energy injected at the inversion, as assumed in the mixed layer model. This happens independently of whether the cloud is being cooled by evaporative or radiative cooling.

The flow in a CBL is characterized by the convective Reynolds number and by the convective Richardson number

$$
\begin{aligned}
& \mathrm{Re}^{*}=z^{*} w^{*} / \nu \simeq\left(z^{*} / \eta\right)^{4 / 3}, \\
& \mathrm{Ri}^{*}=z^{*} \Delta b /\left(w^{*}\right)^{2} .
\end{aligned}
$$

The convective Reynolds number represents the scale separation in the convective boundary layer. The convective Richardson number is the Richardson number associated to the largest eddies of the convective boundary layer. This Richardson number is equivalent to the Richardson number traditionally used in tank experiments $\mathrm{Ri}^{\mathrm{tank}}=H \Delta b /\left(w^{*}\right)^{2}$, where $H$ is the tank depth (Sayler and Breidenthal 1998). Notice that in our experiments, those nondimensional numbers are not constant and grow with the boundary layer depth. The values of these nondimensional numbers at the final time of each simulation are shown in Table 1 . These are relatively large, consistent with the highly turbulent CBL topped by a relatively flat inversion layer observed in Fig. 2 .

Another consequence of the convective scaling is that the flow in the boundary layer is uniquely defined by $z^{*}$

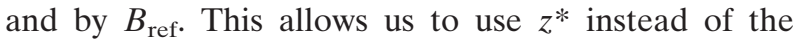
physical time to describe the stage of the system as it is done in the rest of the paper. The main advantage of using this convective scale over the physical time is that $z^{*}$ is independent of the initial transient, allowing us to compare between cases. The initial transient (also called spinup period) lasts roughly $10 t_{0}$ and finishes when the convective length scale is larger than six optical depths $\left(\sim z^{*} / \lambda>6\right)$. After this time flow quantities in the CBL are self-similar as explained above.

\section{b. The inversion layer}

In the previous section we demonstrate that the CBL in the cloud bulk is triggered by $Q_{\mathrm{cbl}}$. Equation (18) 
shows that the rest of the radiative cooling, $Q_{\text {inv }}$, determines the cooling of the inversion layer. The topic of this section is devoted to understand $Q_{\text {inv }}$, and its dependence on the stratification, viscosity, and boundary layer size, as defined by $\mathrm{Ri}_{0}, \mathrm{Re}_{0}$, and $z^{*}$, respectively, in order to allow predictions for atmospheric conditions.

The cooling rate of the inversion layer is divided into two processes by integrating Eq. (8):

$$
-Q_{\text {inv }}=\frac{d z_{i}}{d t} \Delta b+\frac{\partial}{\partial t}\left[\int_{z_{i}}^{\infty}(\Delta b-\langle b\rangle) d z\right] .
$$

This expression states that the total cooling is equal to a term that accounts for the inversion point motion, where $w_{e}=d z_{i} / d t$ is the entrainment velocity, plus a shape term that quantifies the deformation of the cloud interface. This shape term was already identified by Sullivan et al. (1998) in the study of the dry CBL, where they found that its contribution to the inversion balance is not negligible. Although the cloud advancement and deformation are conceptually quite different, the distinction between both processes becomes quite arbitrary in a cloud whose interface deforms quickly. Therefore, deformation and advancement are not studied separately in this paper.

Many of the previous experimental and numerical studies focus on a boundary layer that is bounded by a surface at the bottom. In this bounded configuration, and when the top stratification is high enough, the inversion evolves so slowly that the inversion shape is quasi stationary. In this case it is very likely that the shape term in Eq. (23) can be neglected so that the expression $-Q_{\text {inv }}=w_{e} \Delta b$ is a valid approximation. In this limit different definitions of the inversion point should also provide very similar entrainment velocities, validating this simpler approach for those cases. This is, however, not a valid approximation for a not-bounded boundary layer that grows quickly in time like the one presented in this paper, where all terms in Eq. (23) are comparable.

Figure 7 shows $Q_{\mathrm{inv}}$ as a function of the boundary layer depth for all the cases. The absolute value of $Q_{\text {inv }}$ increases with $z^{*}$ and $\mathrm{Ri}_{0}$ and decreases with increasing $\operatorname{Re}_{0}$. However, $Q_{\text {inv }}$ does not seem to follow any simple functional dependence based on these nondimensional numbers or on the numbers that describe the CBL (Re* and $\left.\mathrm{Ri}^{*}\right)$. This complexity arises because $Q_{\text {inv }}$ is the sum of different mechanisms that cool the inversion. Those mechanisms can be isolated by integrating the horizontally averaged Eq. (8) from the inversion point to infinity:

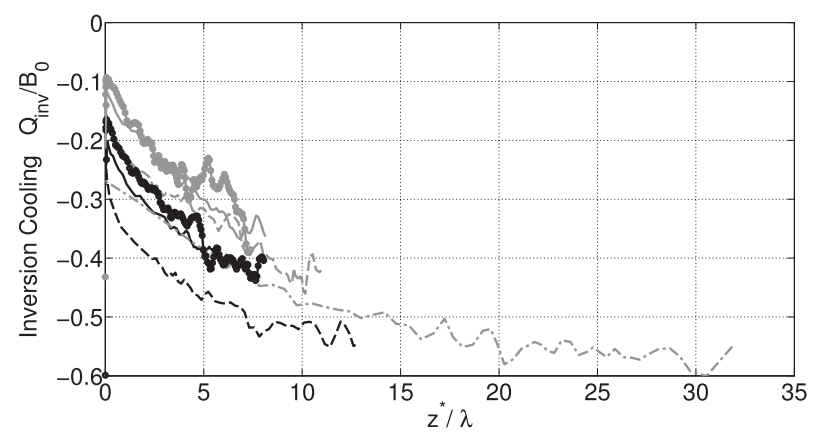

FIG. 7. Rate of change of buoyancy at the inversion as a function of the convective boundary layer length scale. The color codes the Richardson number: black for $\mathrm{Ri}_{0}=57$ and gray for $\mathrm{Ri}_{0}=10$. The type of line codes the Reynolds number: points over lines for $\mathrm{Re}_{0}=$ 1600 , solid for $\mathrm{Re}_{0}=800$, dashed for $\mathrm{Re}_{0}=400$, and dashed-dotted for $\mathrm{Re}_{0}=90$.

$$
\begin{aligned}
Q_{\mathrm{inv}}(t) & =\frac{\partial}{\partial t} \int_{z_{i}}^{\infty}\langle b(z)\rangle d z \\
& =\underbrace{\left\langle w^{\prime} b^{\prime}\right\rangle_{z_{i}}}_{B_{\mathrm{turb}}} \underbrace{-\kappa_{t}\left\langle\frac{\partial b}{\partial z}\right\rangle_{z_{i}}}_{B_{\mathrm{mol}}}-\underbrace{B_{0}\left[1-e^{-\tau\left(z_{i}\right)}\right]}_{B_{\mathrm{dc}}},
\end{aligned}
$$

where the cooling rate at the inversion layer is divided into a turbulent flux $B_{\text {trub }}$, a molecular flux $B_{\text {mol }}$, and the direct cooling of the inversion by radiation $-B_{\mathrm{dc}}$. Notice that this equation is only valid using the previously proposed definition for the inversion point. For any other value of $z_{i}$, a term $w_{e}\left\langle b\left(z_{i}\right)\right\rangle$ appears on the righthand side of the equation. The topic of the next subsections is the independent study of the individual mechanisms that cool the inversion.

\section{1) The turbulent Flux}

The turbulent flux contribution to the inversion cooling $\left\langle w^{\prime} b^{\prime}\right\rangle_{z_{i}}$ accounts for the turbulent exchange of energy between the cloud and the inversion layer. In general dry air is dragged into the cloud, warming the convective boundary layer, while cold air from the cloud enters the inversion. The turbulent flux is usually identified as the main source for energy exchange in stratocumulus clouds. Sayler and Breidenthal (1998) measured $\left|\left\langle w^{\prime} b^{\prime}\right\rangle_{z_{i}}\right|=0.2 B_{0}$ in a tank experiment that mimics a smoke cloud. Similar values are found in the general case of the STBL and dry CBL (Stevens 2002) once an appropriate $B_{0}$ is identified.

Figure 8 shows the turbulent flux as a function of the CBL depth for all our experiments. After the initial transient (or spinup) it falls in the interval $\left\langle w^{\prime} b^{\prime}\right\rangle / B_{0}=$ $-0.175 \pm 0.05$ for all of the cases with $\operatorname{Re}_{0}>90$. Notice that the turbulent flux shown here is measured at a fixed 


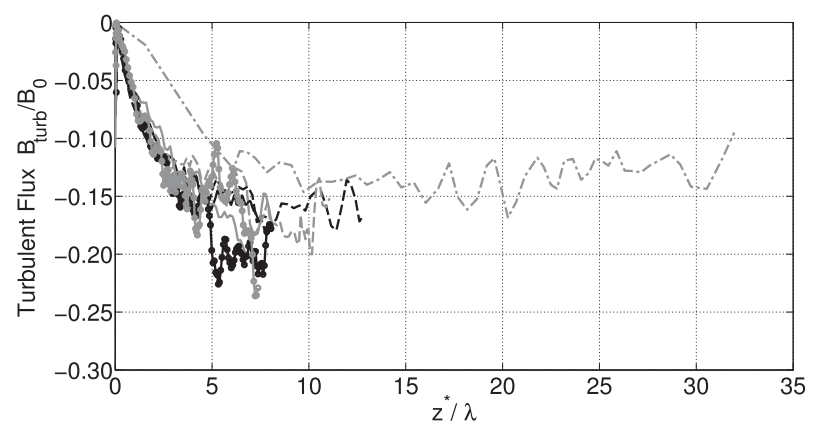

FIG. 8. Turbulent exchange of energy at the inversion point as a function of the convective boundary layer length scale. The color codes the Richardson number: black for $\mathrm{Ri}_{0}=57$ and gray for $\mathrm{Ri}_{0}=10$. The type of line codes the Reynolds number: points over lines for $\mathrm{Re}_{0}=1600$, solid for $\mathrm{Re}_{0}=800$, dashed for $\mathrm{Re}_{0}=400$, and dashed-dotted for $\mathrm{Re}_{0}=90$.

location given by $\langle b\rangle\left(z_{i}\right)=0$. Measurements of turbulent flux at the position of minimum buoyancy (as proposed by other studies) increase the turbulent flux by approximately $10 \%$. The turbulent flux in our simulations is independent of the stratification for the range of Richardson numbers here considered. However, the turbulent flux magnitude increases weakly with the Reynolds number. The entrainment thus increases when smaller Kolmogorov eddies are included, suggesting that the smallest eddies do entrain warm air. These variations of the turbulent flux with $\mathrm{Re}_{0}$ are evident when increasing the reference Reynolds number from 90 to 400 . However, when increasing $\mathrm{Re}_{0}$ from 400 to 1600 the increase of the turbulent flux is very small, suggesting that we are reaching the asymptotic state that is independent of the viscosity.

After the initial transient the turbulent flux is independent of the size of the boundary layer $z *$ for all the stratifications we have considered here $\left(10<\mathrm{Ri}_{0}<57\right)$. For $z^{*}=4-6 \lambda$ all curves level off at $\left\langle w^{\prime} b^{\prime}\right\rangle / B_{0}=-0.175 \pm$ 0.05 , although the flux oscillates owing to the lack of statistical convergence. This means that the turbulent flux does not significantly change by increasing the size of the CBL. We thus conclude that the turbulent flux in our simulations is independent of the largest eddies, which do not seem to contribute to the turbulent entrainment. This is in accordance with the studies of turbulence capped by a strong inversion (Linden 1973; Breidenthal 1992; McGrath et al. 1997; Fernando 1991; and references therein). They observed that for large enough stratifications, only eddies of certain size are able to directly entrain warm air from the inversion. They propose that larger eddies increase the entrainment through secondary processes that generate smallscale motion (internal gravity waves inside the inversion or Kelvin-Helmhotz instability). However, the efficiency of this second mechanism is still under discussion and direct visualizations of our simulations did not show clearly any of those events. Hence, although we cannot completely rule out this mechanism, our results suggest that it does not contribute significantly to the entrainment.

Our results indicate the main contribution to the entrainment comes from eddies of size $z^{*}<4-6 \lambda$. This is only an upper limit for the eddy size because the time to create these eddies coincides with the initial transient of the CBL below the inversion. It is thus likely that the smaller eddies are still evolving until the CBL reaches this size, with direct consequences for the entrainment. As a consequence, we do not know whether eddies of size $z^{*}=4-6 \lambda$ participate for the entrainment but it is clear from Fig. 8 that adding larger eddies does not seem to alter the entrainment values. We hope that further investigations will help to clarify this question.

\section{2) The MOlecular FluX}

The molecular flux quantifies the averaged exchange of heat owing to molecular diffusion between the inversion layer and the convective boundary layer. Its mathematical expression is given by the second term of Eq. (24). Its contribution is expected to be negligible for typical atmospheric Reynolds numbers but this does not need to be the case for the Reynolds numbers we achieve in our simulations.

Figure 9a shows the averaged molecular flux as a function of the convective scale $z^{*}$. For all the simulations with $\operatorname{Re}_{0} \geq 400$, the molecular flux at the inversion point is about one order of magnitude lower than the turbulent flux. We conclude that in a first approximation molecular flux does not play an important role in the exchange of energy between the inversion and the convective boundary layer. However, this does not mean that molecular diffusion can be neglected for the entrainment. Above the inversion point, molecular diffusion grows to much higher values and can even overtake the turbulent flux if the stratification is strong enough (as in the case $\mathrm{Ri}_{0}=57$; not shown here). This has direct consequences for the entrainment in our simulations as we show below.

\section{3) The DIRECT COOLING}

Direct cooling is the part of the radiative cooling that directly cools the inversion layer and it is caused by the smoke that enters into the inversion layer (as shown in Fig. 4). The concept of direct cooling was first introduced by Lilly (1968) and extended by Deardorff (1976) for the parameterization of the entrainment. Lilly and Schubert (1980), Deardorff (1981), Moeng et al. (1999), and Lock and Mac Vean (1999) suggested that the direct 

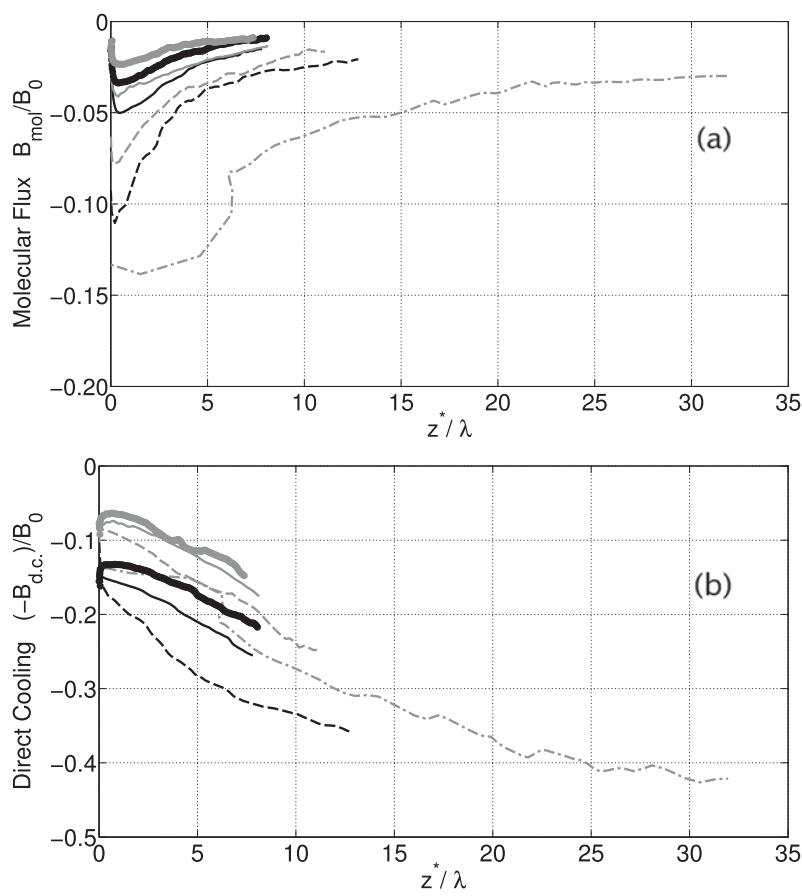

FIG. 9. (a) Molecular diffusion at the inversion point. (b) Direct cooling of the inversion. Both quantities are plotted as a function of the convective boundary layer length scale. The color codes the Richardson number: black for $\mathrm{Ri}_{0}=57$ and gray for $\mathrm{Ri}_{0}=10$. The type of line codes the Reynolds number: points over lines for $\mathrm{Re}_{0}=$ 1600 , solid for $\operatorname{Re}_{0}=800$, dashed for $\operatorname{Re}_{0}=400$, and dashed-dotted for $\mathrm{Re}_{0}=90$.

cooling is indeed an important contribution to the entrainment $(30 \%-60 \%$ of the total entrainment rate) but currently its relevance is still under debate and many authors neglect it completely (Wood 2012). The main uncertainty arises in the definition of the inversion point because the magnitude of the direct cooling is very sensitive to this definition. Using the definition presented here $\left[\langle b\rangle\left(z_{i}\right)=0\right]$, the direct radiative cooling in our simulations [third term of Eq. (24)] makes a major contribution to the cooling of the inversion layer. Figure $9 \mathrm{~b}$ shows that the direct cooling is comparable in magnitude to the turbulent flux, and that in some cases it is the main source for the inversion cooling. Figure $9 \mathrm{~b}$ also shows that the magnitude of the direct cooling decreases with increasing $\mathrm{Re}_{0}$ and that it increases with $z^{*}$ and with $\mathrm{Ri}_{0}$. However, there is no simple relation between the direct cooling and any of the parameters that describe the problem.

To understand the behavior of the direct cooling, it is useful to introduce the inversion-layer thickness (Moeng et al. 1999; Lock and Mac Vean 1999; Lilly 2002). We define the inversion thickness $\delta_{i}$ as the distance from the inversion point to the position where the averaged buoyancy profile reaches $95 \%$ of the dry air

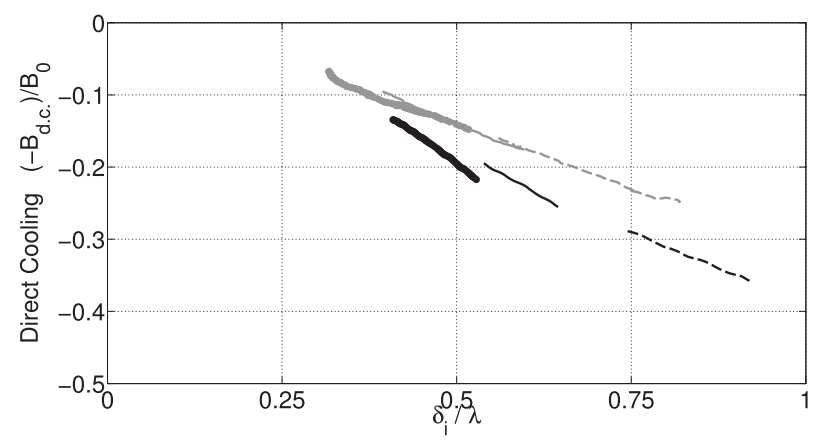

FIG. 10. Direct cooling as a function of the inversion thickness. Notice that all experiments with the same reference Richardson number (coded by color) align to the same line, so that the Reynolds number dependency (coded by line style) drops. Black represents $\mathrm{Ri}_{0}=57$ and gray $\mathrm{Ri}_{0}=10$. Points over lines are used for $\mathrm{Re}_{0}=1600$, solid for $\mathrm{Re}_{0}=800$, and dashed for $\mathrm{Re}_{0}=400$.

buoyancy. This definition is based over the mean profile and therefore it is a global measure. In Fig. 10 we show the direct cooling as a function of the ratio of the inversion thickness over the optical depth. As the inversion layer broadens, the amount of smoke inside the inversion layer increases, which in turn increases the direct cooling. The optical depth scales how the variations of the inversion thickness are translated into changes in the radiative forcing. For the same ratio $\delta_{i} / \lambda$, we observe that the direct cooling magnitude increases with the stratification as given by the reference Richardson number. This tendency is explained by looking at the mixture of smoke and dry air at the inversion point. The smoke-air mixture is less diluted for the higher Richardson numbers for which the mixing with the free atmosphere is less efficient. A smoke-rich mixture increases the amount of smoke at the inversion and therefore the direct cooling. Remarkably all experiments for the same stratification number align for different reference Reynolds numbers. Assuming that this tendency will hold for higher Reynolds number, it is then possible to use Fig. 10 to extrapolate direct cooling values to atmospheric conditions when the inversion thickness and optical depth are known.

The tendencies observed in Fig. 9 are explained in terms of the ratio $\delta_{i} / \lambda$. For example, the viscosity dependence of the direct cooling is mainly an effect of the inversion broadening due to the molecular diffusion in our system. By increasing $\mathrm{Re}_{0}$, the inversion becomes thinner, giving space to less smoke inside the inversion layer and reducing the direct cooling. The $z^{*}$ dependence is a result of the deformation of the inversion layer caused by the turbulent convective boundary layer. As larger and larger eddies hit the inversion, the inversion becomes convoluted and its mean profile 
thickens, which results in less mean molecular flux and higher direct cooling. All eddies contribute to the inversion deformation but the largest, with more kinetic energy, are the most efficient ones for this process.

\section{4) THE INVERSION-LAYER THICKNESS}

We have shown in Fig. 10 that the direct cooling is given by the ratio of inversion-layer thickness over the optical depth $\delta_{i} / \lambda$ and by the stratification. It is left to understand how this ratio evolves for different flow conditions. Here we consider two limiting cases.

In the first limiting case we assume that $\mathrm{Re}_{0}$ is low enough for molecular diffusion to dominate but such that $\delta_{i} / \lambda<1$, so that the inversion does not spreads over several optical depths and molecular diffusion adsorbs all radiative cooling. Under these conditions, entrainment is dictated by radiative cooling and not by the molecular diffusion. Following literature values we assume a typical entrainment of $0.2 B_{0}$. In the absence of turbulent transport, molecular diffusion adjusts the inversion thickness so that the molecular flux dominates the inversion: $\kappa_{t}(\partial b / \partial z) \sim 0.2 B_{0}$. The resulting inversion thickness can be then estimated for $\operatorname{Pr}=1$ as

$$
\delta_{i}^{\mathrm{mol}} / \lambda=\frac{5 \kappa_{t} \Delta b}{\lambda B_{0}}=5 \mathrm{Ri}_{0} / \operatorname{Re}_{0} .
$$

In the second limiting case we assume negligible molecular diffusion in the inversion layer $\left(\operatorname{Re}_{0} \rightarrow \infty\right)$. Therefore the cloud top at each position is infinitely thin. However, the same is not true for the averaged profile, which presents a finite thickness when the cloud top is convoluted. This thickness is then determined by large turbulent eddies of the convective boundary layer that hit and deform the cloud top. In the previous sections we characterized velocity of the largest eddies with the convective velocity $w^{*}$. A simple balance between kinetic and potential energy suggests that a Richardson number of the kind $\Delta b \delta_{i}^{\text {turb }} /\left(w^{*}\right)^{2}$ should be of order one. Lock (1998) already proposed this balance for stratocumuli and the resulting inversion-layer thickness is comparable to the ones measured by tethered balloon soundings (Haman 2009). Using this balance the resulting thickness is

$$
\delta_{i}^{\mathrm{turb}} / \lambda=\left(z^{*} / \lambda\right) / \mathrm{Ri}^{*},
$$

where we have written $\delta_{i}^{\text {turb }}$ as a function of the convective scales in order to stress that the inversion thickness in this limit is only determined by the large-size eddies. In our simulations we find that the ratio $\delta_{i} / \delta_{i}^{\text {turb }}$ varies strongly from case to case, which indicates that molecular diffusion is still playing an important role.
However, in all simulations with $\mathrm{Ri}_{0}=10, \delta_{i} / \delta_{i}^{\text {turb }}$ levels to a constant value from $z^{*} \sim 4 \lambda$, suggesting that $\delta_{i}^{\text {turb }}$ is indeed the relevant length for the inversion thickness in the limit of negligible molecular flux. For the cases of lowest molecular diffusion $\left(\mathrm{Re}_{0}=800, \mathrm{Ri}_{0}=10\right)$ and $\left(\mathrm{Re}_{0}=1600, \mathrm{Ri}_{0}=10\right)$ we found $\delta_{i} / \delta_{i}^{\text {turb }} \simeq 1.4$, which is consistent with the hypothesis presented above.

Both inversion thicknesses, $\delta_{i}^{\text {turb }}$ and $\delta_{i}^{\text {mol }}$, provide the limiting value of the inversion thickness in the limits of weak and strong molecular diffusion. The ratio

$$
\mathrm{Ri}_{(i)}=\delta_{i}^{\mathrm{mol}} / \delta_{i}^{\mathrm{turb}}=5\left(\mathrm{Ri}^{*}\right)^{2} / \mathrm{Re}^{*}
$$

provides a measure of the importance of the molecular diffusion for the inversion thickness. This ratio is the internal Richardson number, as defined by McGrath et al. (1997), when the inversion thickness is $\delta_{i}=\delta_{i}^{\mathrm{mol}}$. For $\operatorname{Ri}_{(i)} \gg 1$, the inversion thickness is determined solely by molecular diffusion and for $\operatorname{Ri}_{(i)} \ll 1$, molecular diffusion can be completely neglected. Notice that as the CBL grows the value of $\mathrm{Ri}_{(i)}$ decreases and a change of regime might occur depending of the values of viscosity and stratification. In the simulations of the strong stratification $\left(\mathrm{Ri}_{0}=57\right)$ we found $\mathrm{Ri}_{(i)}>3$, which means that the inversion thickness is mainly governed by molecular effects. The lower stratifications are characterized by $\operatorname{Ri}_{(i)}<0.25$ when the CBL reaches its maximum extension, which means that the inversion thickness is mainly determined by the large convective eddies. However, in all cases $\operatorname{Ri}_{(i)}$ is of order unity, which means that neither molecular diffusion nor turbulence can be neglected.

\section{5) THE ENTRAINMENT OF DRY AIR}

In previous sections we have concentrated on the exchange of energy between the cloud bulk and the inversion but in many applications it is also of crucial importance to know how much air from the free atmosphere is entrained at the cloud top. We study the variation of dry air $1-f$ in the free atmosphere, given by

$$
S_{\text {inv }}(t)=\partial\left[\int_{z_{i}}^{\infty}(1-\langle f\rangle) d z\right] / \partial t .
$$

In some zero-order models (Stevens 2002) it is assumed that the flux into the cloud is the same for all of the scalars and therefore the scaled flux of buoyancy is equal to the scaled flux of dry air. However, this simple idea does not apply to our results even in the cases where molecular effects are negligible. For example, in Fig. 4 the scaled turbulent smoke flux is clearly higher than the turbulent buoyancy flux. Instead, the variation of smoke at the inversion is much closer to the scaled total 


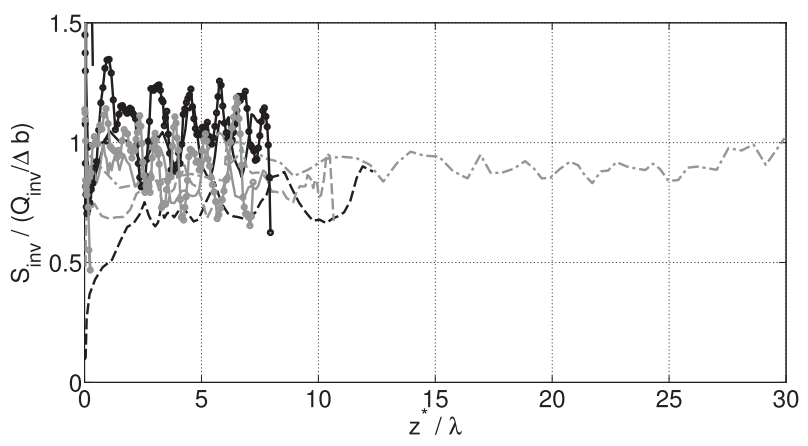

FIG. 11. Flux of noncloudy air into the cloud divided by the change of buoyancy in the cloud. The color codes the Richardson number: black for $\mathrm{Ri}_{0}=57$ and gray for $\mathrm{Ri}_{0}=10$. The type of line codes the Reynolds number: points over lines for $\mathrm{Re}_{0}=1600$, solid for $\mathrm{Re}_{0}=800$, dashed for $\mathrm{Re}_{0}=400$, and dashed-dotted for $\mathrm{Re}_{0}=90$.

variation of buoyancy at the inversion, $Q_{\text {inv }} / \Delta b$, as shown in Fig. 11. The dry air entrainment thus follows

$$
S_{\text {inv }}(t)=C_{s} Q_{\text {inv }}(t) / \Delta b,
$$

where $C_{s}$ is a numerical constant that can be approximated as $C_{s}=1.0 \pm 0.2$. The consequence is that all inversion cooling (including the direct cooling) is translated into the mixing of the cloud smoke. Our results are thus close to the limit $C_{s}=1.0$, which is a condition for the synchronized motion of the smoke and buoyancy profiles at the inversion.

\section{Relevance for atmospheric studies}

In previous sections we investigated $Q_{\text {inv }}$ and its dependence on the flow dynamics. The inversion cooling does not only provide the inversion-layer deformation and displacement but also the buoyancy flux into the cloud-bulk CBL, which is calculated using the energy conservation constraint $Q_{\mathrm{cbl}}=-B_{0}-Q_{\text {inv }}$. In this section we propose parameterizations for $Q_{\text {inv }}$ for atmospheric conditions and for LES.

\section{a. Comparison with atmospheric measurements}

In the light of the results presented in this paper we provide new estimations for entrainment at high Reynolds numbers and typical stratocumulus stratifications, when evaporative effects, shear, and microphysical effects are neglected. Recall that the inversion layer in our simulations is cooled by the combined action of direct radiative cooling and molecular and turbulent fluxes. We have thus to provide estimates for all these processes at high Re. High Re allows us to neglect the molecular flux. Our simulations show that the turbulent flux does not depend appreciably on the Reynolds number. We thus expect that the turbulent entrainment for very high $\mathrm{Re}$ is similar to the simulation value $\left\langle w^{\prime} b^{\prime}\right\rangle_{z_{i}}=-0.175 B_{0}$. To finish, the direct cooling is given by the ratio of the inversion-layer thickness to the optical depth. In particular, the extrapolation of the direct cooling in Fig. 10 for the stratification that mimics the atmospheric case $\left(\mathrm{Ri}_{0}=57\right)$ yields $B_{\mathrm{dd}} / B_{0} \simeq-0.39 \delta_{i} / \lambda$ (note that this expression is only valid for $\delta_{i} / \lambda<1$ ). Once the inversion cooling is known, the entrainment velocity is calculated using Eq. (23) and neglecting the shape term in the righthand side of that equation,

$$
w_{e}=\frac{F_{0}}{\rho c_{p} \Delta T}\left(0.175+0.39 \delta_{i} / \lambda\right)
$$

where the first contribution is due to the turbulent flux and the second is due to the direct cooling. Further, in the atmospheric limit molecular diffusion is neglected $\left[\mathrm{Ri}_{(i)} \ll 1\right.$ as discussed in section 5] and the inversion-layer thickness is determined by a simple balance of kinetic and potential energy, which yields $\delta_{i}^{\text {turb }} / \lambda \simeq\left(w^{*}\right)^{2} /(\lambda \Delta b)$. The resulting parameterization for atmospheric clouds reads

$$
w_{e}=\frac{F_{0}}{\rho c_{p} \Delta T}\left[0.175+0.39 \frac{\left(w^{*}\right)^{2} T_{c}}{\lambda g \Delta T}\right] .
$$

Numerical estimates of entrainment can be given using Eq. (31) and the radiation parameters presented by Larson et al. (2007) for the flight RF01 of the DYCOMS II campaign $\left(\lambda=15 \mathrm{~m}, F_{0}=62 \mathrm{~W} \mathrm{~m}^{-2}\right.$, and $\left.B_{0}=1.9 \times 10^{-3} \mathrm{~m}^{2} \mathrm{~s}^{-3}\right)$. The resulting radiative direct cooling lies in the interval $0.05<B_{\mathrm{dc}} / B_{0}<0.2$, the total inversion cooling lies in the interval $0.2<\left|Q_{\text {inv }}\right| / B_{0}<$ 0.4 , and total cloud-bulk cooling is bounded by $0.6<$ $\left|Q_{\mathrm{cbl}}\right| / B_{0}<0.8$. For a boundary layer of size $z_{i}=$ $800 \mathrm{~m}$, the velocity fluctuations are estimated as $w^{*}=$ $\left(Q_{\mathrm{cbl}} z_{i}\right)^{1 / 3}=1.0 \pm 0.2 \mathrm{~m} \mathrm{~s}^{-1}$. This value is in accordance with the typical velocities measured in the nocturnal flights of the DYCOMS II campaign. The entrainment velocities calculated from Eq. (31) are compared in Fig. 12 to the measurements from different flights in the same campaign (Faloona et al. 2005; Gerber et al. 2005). The figure shows that our estimation provides a lower bound for the entrainment velocities and follows the same tendency with the stratification as the measurements. Radiative cooling thus accounts for a large part of the entrainment (between $50 \%$ and $100 \%$ of the total entrainment), although this value might be overestimated by our model approximations as discussed below. The discrepancy with the cloud values suggests that shear, evaporative cooling or other physical phenomena increase the entrainment rate over that induced by radiative cooling alone. 


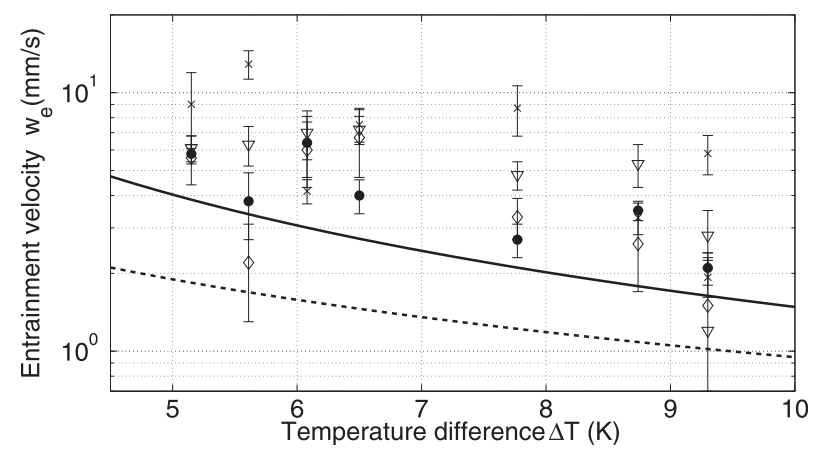

FIG. 12. The entrainment velocity measured in the DYCOMS II campaign as a function of the stratification (Faloona et al. 2005; Gerber et al. 2005). The symbols represent the method that was used for measuring the entrainment: squares for ozone, circles for DMS, triangles for water, and crosses for the hole's method. The continuous line represents our estimation using Eq. (31) and the radiation parameters given by Larson et al. (2007) for the flight RF01 of the same campaign. The dashed line corresponds to the same estimation, but neglecting the direct cooling.

Next, we discuss how the entrainment is modified by our two main simplifications: the smoke cloud and the one-dimensional horizontally averaged radiation model. Contrary to a smoke, cloud liquid water evaporates at the cloud interface. As a result, it is likely that the concentration of liquid water in the inversion layer is lower than the concentration of smoke in the dry model. If this is the case, the direct cooling is overestimated in the smoke approximation. This effect adds to the deviations by the averaged radiation model. This radiation model cools smoke-free parcels when the cloud interface is convoluted (see section 3), probably increasing the direct cooling. As a consequence, the second term in Eq. (31) can be understood as an upper bound for the direct cooling, providing a reference for future simulations with more complex models.

To estimate how much the direct cooling in stratocumuli deviates from our calculations, we plot in Fig. 12 the entrainment velocity using Eq. (31) but completely neglecting the direct cooling. This estimate provides much lower entrainment velocities than the measurements, suggesting the important role of the direct cooling in the atmospheric context.

\section{b. Comparison with LES}

Our results indicate that a very high resolution is required at the inversion in order to obtain reliable entrainment rates, confirming previous studies (Moeng et al. 1996; Stevens 2002; Bretherton et al. 1999; Heus et al. 2010). If, as our results suggest, the turbulent flux is performed by eddies of size smaller than approximately four optical depths $(\sim 60 \mathrm{~m})$, models that do not capture those eddies rely uniquely on subgrid schemes and numerical diffusion. We also show that probably even a higher resolution is necessary to represent the inversion dynamics correctly, including the direct cooling. Typical numerical treatment of diffusion in LES models introduce numerical diffusion near the sharp gradients in temperature and moisture that occur at the inversion. Such numerical diffusion avoids spurious extremes (over- and undershoots) in the temperature and humidity fields but is often applied more broadly, leading to an overestimate of the inversion thickness. This inversion broadening results in an undesired increase of the direct cooling of the inversion.

One of the findings of the present paper is the proper determination of the inversion point in an unbounded mixed layer $\left[\langle b\rangle\left(z_{i}\right)=0\right]$. However, it is not trivial how to extend this definition of the inversion point to LES of the STBL because LES mean buoyancy profiles do not show the buoyancy minimum close to the inversion that is required for this definition. We speculate that the minimum is not reproduced by LES either because LES do not accurately capture the buoyancy finescale structure, or because the minimum is blurred by the largescale circulation. From the alternative choices of the inversion point we find that the minimum turbulent flux best reproduces the CBL scalings presented in section 5 . This point is close to our inversion point for all of the cases presented here, and the contributions to the energy inversion balance deviate by only about $10 \%$ from the ones presented in this paper. Choosing the maximum gradient of the buoyancy as the inversion point does not reproduce the CBL scalings presented here at all.

We compare our results with the smoke LES intercomparison of Bretherton et al. (1999). In that study, the authors performed simulations of a semi-infinite smoke cloud with a very large optical depth $(\lambda=45 \mathrm{~m})$. The reference Richardson number in that study $\mathrm{Ri}_{0}=58$ is very close to our simulations with the strong stratification. They found that the inversion-layer thickness is $\delta_{i}=0.45 \lambda$ and $\delta_{i}=1.1 \lambda$, for the, at that time, high (5 m)and the normal $(25 \mathrm{~m})$-vertical-resolution simulations, respectively. These values are much higher than the estimation $\delta_{i}^{\text {turb }}=0.1 \lambda$ that is obtained in the limit $\operatorname{Re}_{0} \rightarrow \infty$ using Eq. (26). The strong discrepancy between the LES and our estimate is probably due to numerical diffusion or due to the subgrid model.

We can also compare the entrainment velocities between the LES results and our prediction. Using the probably-too-high inversion thicknesses measured in Bretherton et al. (1999), Eq. (30) provides $w_{e}=$ $2.6 \mathrm{~mm} \mathrm{~s}^{-1}$ and $w_{e}=4.5 \mathrm{~mm} \mathrm{~s}^{-1}$ for the high and normal resolution. Remarkably, Eq. (30) gives the same velocity as measured by Bretherton et al. (1999) in the 
high-resolution case, and overestimates the normalresolution entrainment velocity by just $25 \%$. This agreement confirms the here-proposed form for the direct cooling, Fig. 10, and indicates that the main cause for the high entrainment velocities in the LES by Bretherton et al. (1999) is the inversion broadening by numerical diffusion or by the subgrid model. This problem is probably still unresolved in contemporary LES (Yamaguchi and Randall 2012), which use higher resolutions than Bretherton et al. (1999), but still much lower than in the present study. Based on the condition on the internal Richardson number [Eq. (27)], we fear that a much higher resolution (on the order of $1 \mathrm{~cm}$, but depending on the numerical and subgrid scheme) will be necessary to completely eliminate the enhancement of direct cooling by diffusion in LES or DNS. This prediction is only valid for the cases with strong stratifications $(\sim 8 \mathrm{~K})$ and little shear.

\section{c. The liquid water feedback and aerosols}

In this paper we have identified that the direct cooling seems to play an important role for the entrainment in stratocumuli, as previously suggested by Deardorff (1976), Lock (1998), and Moeng et al. (1999). We can then consider the main consequences of the direct cooling for the stratocumulus long time dynamics. In particular, we concentrate on the consequences of varying the optical length for the direct cooling. According to our results, the direct cooling always adds to the entrainment and increases as the optical length becomes smaller. This result was observed in the limit $\delta_{i} / \lambda<1$.

First, the optical length is reduced by increasing the liquid water concentration in the cloud [see Eqs. (2) and (5)]. As a consequence, any increase of the liquid water in the entrainment zone amplifies the entrainment via the direct cooling [see Eq. (31)]. Enhanced entrainment decreases the liquid water concentration until an equilibrium is reached. We can then conclude that the direct cooling constitutes a negative feedback for the liquid water content. This negative feedback is similar to the one proposed by Moeng et al. (1995).

Second, the optical length is also sensitive to the effective radius of the droplets. Decreasing the effective radius (e.g., by adding aerosols) reduces the optical length, which in turn increases the direct cooling and the entrainment. This effect adds to the enhancement of the entrainment by decreasing sedimentation when the droplet radius is reduced (Ackerman et al. 2004). Therefore clouds with higher droplet concentrations would present more entrainment and, in absence of drizzle, would break more easily than the same clouds with fewer droplets.

\section{Summary and conclusions}

We have investigated a cloud-top mixing layer that is driven by radiative cooling using direct numerical simulations. The complexity of the problem is reduced by applying two main simplifications: the radiation is calculated using a one-dimensional horizontally averaged model, and there is no evaporative cooling. This simplified configuration mimics one relevant aspect of the cloud top in a stratocumulus-topped boundary layernamely, the mixing across an inversion that bounds a radiatively driven turbulent flow. The problem can be defined in terms of only two parameters: a reference Reynolds number and the stratification strength (a reference Richardson number). For this study we choose a reference stratification that corresponds to a temperature jump $\Delta T \sim 9.5-11 \mathrm{~K}$ as measured in the flight RF01 in the DYCOMS II field campaign (Stevens et al. 2003a). We also investigate one weaker stratification in order to evaluate the importance of the stratification strength for the flow. For each stratification we investigate different Reynolds numbers to assess the effect of molecular diffusion.

The initial condition is set such that the cloud is at constant temperature with very weak turbulence. As radiation cools the top of the cloud, a convective boundary layer (CBL) grows downward in the cloud. This configuration differs from previous studies where the boundary layer extends down to the surface. The main advantage of our configuration is that the integral scale (the size of the largest eddies) grows continuously. This allows us to investigate the effect of diverse scales on the entrainment.

\section{Our main conclusions are as follows:}

- By defining the inversion point by $\langle b\rangle\left(z_{i}\right)=0$, we derive an exact energy balance equation that divides the total radiative cooling $B_{0}$ into two components: the cooling of the cloud $Q_{\mathrm{cbl}}$ and the cooling of the inversion layer $Q_{\text {inv }}$ (see Fig. 13). The inversion cooling has two main contributions: the turbulent flux and the direct cooling.

- Turbulence statistics inside the cloud bulk only depend on $Q_{\mathrm{cbl}}$ and on the size of the generated CBL and they are independent of viscosity, stratification, and whether the cloud is cooled by radiative or evaporative cooling. This clear decoupling of the cloud-bulk turbulence from the inversion layer serves as a justification for mixed-layer models.

- The turbulent flux contribution to the inversion cooling reaches a constant value $\left\langle w^{\prime} b^{\prime}\right\rangle_{z_{i}}=-(0.175 \pm 0.05) B_{0}$ independently of the stratification and viscosity. The turbulent flux levels when the size of the largest eddies in the CBL is around $60 \mathrm{~m}$ (or four optical depths), 


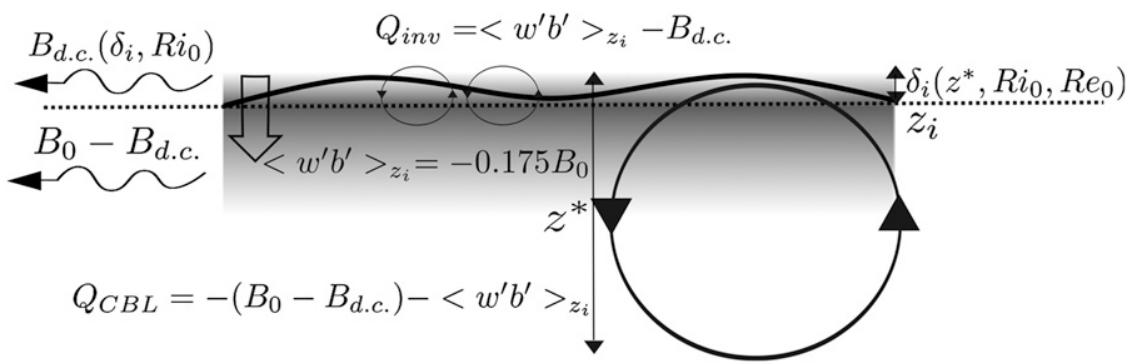

FIG. 13. Energy balance at the inversion. The total radiative cooling $B_{0}$ is divided between the cooling of the CBL in the cloud bulk $Q_{\mathrm{cbl}}$ and the cooling employed for the mixing with the free atmosphere $Q_{\text {inv }}$. The energy balance at the inversion point $z_{i}$ provides the mechanisms that cool the inversion: the turbulent flux $\left\langle w^{\prime} b^{\prime}\right\rangle_{z_{i}}$ and the direct cooling $B_{\mathrm{dc}}$. The turbulent flux reaches a constant value for all our simulations but the direct cooling shows a complex behavior owing to its dependence on the inversion thickness $\delta_{i}$.

suggesting that only eddies smaller than this size participate in the entrainment.

- A nonturbulent contribution to the inversion cooling, the direct cooling, cannot be neglected. We found that its strength solely depends on the ratio of inversion thickness over the optical length and on the stratification. We provide graphically this relation in Fig. 10. This allows us to make predictions of the direct cooling and entrainment velocities for atmospheric conditions and for LES. Any kind of diffusion (numerical, molecular, or subgrid) tends to increase the inversion thickness, which in turn increases the direct cooling. This might explain, at least partially, why LES tends to produce too-large entrainment values in stratocumuli.

- We propose a parameterization in Eq. (31) for the entrainment velocity in atmospheric conditions, when evaporative cooling and shear are neglected. The parameterization combines the effect of the turbulent flux and the direct cooling. The resulting entrainment velocities account for a large part (between $50 \%$ and $100 \%$ ) of the entrainment velocities measured in the DYCOMS II campaign.

Acknowledgments. We thank Robert Pincus for scientific discussions and his advice for the radiation model. Support from the Max Planck Society through its Max Planck Research Groups program is gratefully acknowledged. Computational time was provided by the Jülich Supercomputer Centre.

During the reviewing process, one of the referees made us aware of the unpublished very-high-resolution (horizontal: $5 \mathrm{~m}$; vertical: $1 \mathrm{~m}$ ) LES of the smoke cloud performed by M. Khairoutdinov. As in our paper, Khairoutdinov's results reveal the importance of the direct cooling for the entrainment and show that the turbulent flux at the inversion is independent of the large-scale flow. These LES show the same general tendencies as presented here but in a surface bounded CBL, confirming that our conclusions are strong and general for other configurations.

\section{REFERENCES}

Ackerman, A. S., M. P. Kirkpatrick, D. E. Stevens, and O. B. Toon, 2004: The impact of humidity above stratiform clouds on indirect aerosol climate forcing. Nature, 432, 1014-1017.

— , and Coauthors, 2009: Large-eddy simulations of a drizzling, stratocumulus-topped marine boundary layer. Mon. Wea. Rev., 137, 1083-1110.

Bony, S., J.-L. Dufresne, H. L. Treut, J.-J. Morcrette, and C. Senior, 2004: On dynamic and thermodynamic components of cloud changes. Climate Dyn., 22, 71-86.

Breidenthal, R. E., 1992: Entrainment at thin stratified interfaces: The effects of Schmidt, Richardson, and Reynolds numbers. Phys. Fluids, 4A, 2141, doi:10.1063/1.858510.

Bretherton, C. S., and Coauthors, 1999: An intercomparison of radiatively driven entrainment and turbulence in a smoke cloud, as simulated by different numerical models. Quart. J. Roy. Meteor. Soc., 125, 391-423.

_ tation, entrainment efficiency, and subtropical stratocumulus albedo. Geophys. Res. Lett., 34, L03813, doi:10.1029/ 2006GL027648.

Caldwell, P., C. S. Bretherton, and R. Wood, 2005: Mixed-layer budget analysis of the diurnal cycle of entrainment in southeast Pacific stratocumulus. J. Atmos. Sci., 62, 3775-3791.

Carpenter, M. H., and C. A. Kennedy, 1994: Fourth-order 2N-storage Runge-Kutta schemes. NASA Langley Research Center Tech. Rep. TM-109112, 24 pp.

— D. Gottlieb, and S. Abarbanel, 1993: The stability of numerical boundary treatments for compact high-order finitedifference schemes. J. Comput. Phys., 108, 272-295.

Chillà, F., and J. Schumacher, 2012: New perspectives in turbulent Rayleigh-Bénard convection. Eur. Phys. J., 35E, 58, doi:10.1140/ epje/i2012-12058-1.

Deardorff, J. W., 1970a: Convective velocity and temperature scales for the unstable planetary boundary layer and for Rayleigh convection. J. Atmos. Sci., 27, 1211-1213.

, 1970b: Preliminary results from numerical integrations of the unstable planetary boundary layer. J. Atmos. Sci., 27, 1209-1211. 
1976: On the entrainment rate of a stratocumulus-topped mixed layer. Quart. J. Roy. Meteor. Soc., 102, 563-582.

- 1981: On the distribution of mean radiative cooling at the top of a stratocumulus-capped mixed layer. Quart. J. Roy. Meteor. Soc., 107, 191-202.

Dietze, E., J. P. Mellado, B. Stevens, and H. Schmidt, 2012: Study of low-order numerical effects in the two-dimensional cloudtop mixing layer. Theor. Comput. Fluid Dyn., 27, 239-251, doi:10.1007/s00162-012-0263-0.

Faloona, I., and Coauthors, 2005: Observations of entrainment in eastern Pacific marine stratocumulus using three conserved scalars. J. Atmos. Sci., 62, 3268-3285.

Fedorovich, E., R. Conzemius, and D. Mironov, 2004: Convective entrainment into a shear-free, linearly stratified atmosphere: Bulk models reevaluated through large eddy simulations. J. Atmos. Sci., 61, 281-295.

Fernando, H. J. S., 1991: Turbulent mixing in stratified fluids. Annu. Rev. Fluid Mech., 23, 455-493.

Flack, K. A., J. R. Saylor, and G. B. Smith, 2001: Near-surface turbulence for evaporative convection at an air/water interface. Phys. Fluids, 133, 3338, doi:10.1063/1.1410126.

Gerber, H., G. Frick, S. P. Malinowski, J.-L. Brenguier, and F. Burnet, 2005: Holes and entrainment in stratocumulus. J. Atmos. Sci., 62, 443-459.

Haman, K. E., 2009: Simple approach to dynamics of entrainment interface layers and cloud holes in stratocumulus clouds. Quart. J. Roy. Meteor. Soc., 135, 93-100.

Hartmann, D. L., and D. A. Short, 1980: On the use of earth radiation budget statistics for studies of clouds and climate. J. Atmos. Sci., 37, 1233-1250.

Heus, T., and Coauthors, 2010: Formulation of the Dutch Atmospheric Large-Eddy Simulation (DALES) and overview of its applications. Geosci. Model Dev., 3, 415-444.

Kurowski, M. J., S. P. Malinowski, and W. W. Grabowski, 2009: A numerical investigation of entrainment and transport within a stratocumulus-topped boundary layer. Quart. J. Roy. Meteor. Soc., 135, 77-92.

Larson, V. E., K. E. Kotenberg, and N. B. Wood, 2007: An analytic longwave radiation formula for liquid layer clouds. Mon. Wea. Rev., 135, 689-699.

Lele, S. K., 1992: Compact finite difference schemes with spectrallike resolution. J. Comput. Phys., 103, 16-42.

Lewellen, D. C., and W. S. Lewellen, 1998: Large-eddy boundary layer entrainment. J. Atmos. Sci., 55, 2645-2665.

Lilly, D. K., 1968: Models of cloud-topped mixed layers under a strong inversion. Quart. J. Roy. Meteor. Soc., 94, 292309.

— 2002: Entrainment into mixed layers. Part I: Sharp-edged and smoothed tops. J. Atmos. Sci., 59, 3340-3352.

- , and W. H. Schubert, 1980: The effects of radiative cooling in a cloud-topped mixed layer. J. Atmos. Sci., 37, 482487.

Linden, P. F., 1973: The interaction of a vortex ring with a sharp density interface: A model for turbulent entrainment. J. Fluid Mech., 60, 467-480.

Lock, A., 1998: The parametrization of entrainment in cloudy boundary layers. Quart. J. Roy. Meteor. Soc., 124, 27292753.

—_, and M. K. Mac Vean, 1999: The parametrization of entrainment driven by surface heating and cloud-top cooling. Quart. J. Roy. Meteor. Soc., 125, 271-299.

McEwan, A. D., and G. W. Paltridge, 1976: Radiatively driven thermal convection bounded by an inversion-A laboratory simulation of stratus clouds. J. Geophys. Res., 81, 10951102.

McGrath, J. L., H. J. S. Fernando, and J. C. R. Hunt, 1997: Turbulence, waves and mixing at shear-free density interfaces. 2. Laboratory experiments. J. Fluid Mech., 347, 235-261.

Mellado, J. P., 2010: The evaporatively driven cloud-top mixing layer. J. Fluid Mech., 660, 5-36.

— of the pressure-Poisson equation using finite differences in colocated grids. Z. Angew. Math. Mech., 92, 380-392.

- B. Stevens, H. Schmidt, and N. Peters, 2009: Buoyancy reversal in cloud-top mixing layers. Quart. J. Roy. Meteor. Soc., 135, 333-365.

,,--- , and,- 2010 : Two-fluid formulation of the cloud-top mixing layer for direct numerical simulation. Theor. Comput. Fluid Dyn., 24, 511-536.

Moeng, C., and U. Schumann, 1991: Composite structure of plumes in stratus-topped boundary layers. J. Atmos. Sci., 48, 22802291.

- D. H. Lenschow, and D. A. Randall, 1995: Numerical investigations of the roles of radiative and evaporative feedbacks in stratocumulus entrainment and breakup. J. Atmos. Sci., 52, 2869-2883.

— topped planetary boundary layer: Intercomparison among different numerical codes. Bull. Amer. Meteor. Soc., 77, 261-278.

_ P. P. Sullivan, and B. Stevens, 1999: Including radiative effects in an entrainment rate formula for buoyancy-driven PBLs. J. Atmos. Sci., 56, 1031-1049.

- B. Stevens, and P. P. Sullivan, 2005: Where is the interface of the stratocumulus-topped PBL? J. Atmos. Sci., 62, 26262631.

Moin, P., and K. Mahesh, 1998: Direct numerical simulation: A tool in turbulence research. Annu. Rev. Fluid Mech., 30, $539-578$.

Petterssen, S., 1938: On the causes and the forecasting of the California fog. Bull. Amer. Meteor. Soc., 19, 49-55.

Petty, G. W., 2006: A First Course in Atmospheric Radiation. Sundog Publishing, 472 pp.

Pincus, R., and B. Stevens, 2009: Monte Carlo spectral integration: A consistent approximation for radiative transfer in large eddy simulations. J. Adv. Model. Earth Syst., 1, 1, doi:10.3894/ JAMES.2009.1.1.

Pope, S. B., 2000: Turbulent Flows. Cambridge University Press, $771 \mathrm{pp}$.

Sayler, B. J., and R. E. Breidenthal, 1998: Laboratory simulations of radiatively induced entrainment in stratiform clouds. J. Geophys. Res., 103 (D8), 8827-8837.

Siems, S. T., and C. S. Bretherton, 1992: A numerical investigation of cloud-top entrainment instability and related experiments. Quart. J. Roy. Meteor. Soc., 118, 787-818.

Stephens, G. L., 1978: Radiation profiles in extended water clouds. II: Parameterization schemes. J. Atmos. Sci., 35, 2123-2132.

Stevens, B., 2002: Entrainment in stratocumulus-topped mixed layers. Quart. J. Roy. Meteor. Soc., 128, 2663-2690.

, 2005: Atmospheric moist convection. Annu. Rev. Earth Planet. Sci., 33, 605-643.

_ C. Moeng, and P. P. Sullivan, 1999: Large-eddy simulations of radiatively driven convection: Sensitivities to the representation of small scales. J. Atmos. Sci., 56, 3963-3984. 
_ , and Coauthors, 2003a: Dynamics and chemistry of marine stratocumulus-DYCOMS II. Bull. Amer. Meteor. Soc., 84, 579-593.

- and Coauthors, 2003b: On entrainment rates in nocturnal marine stratocumulus. Quart. J. Roy. Meteor. Soc., 129, 3469 3493.

- and Coauthors, 2005: Evaluation of large-eddy simulations via observations of nocturnal marine stratocumulus. Mon Wea. Rev., 133, 1443-1462.
Sullivan, P. P., C. Moeng, B. Stevens, D. H. Lenschow, and S. D. Mayor, 1998: Structure of the entrainment zone capping the convective atmospheric boundary layer. J. Atmos. Sci., 55, 3042-3064.

Wood, R., 2012: Stratocumulus clouds. Mon. Wea. Rev., 140, 2373 2423.

Yamaguchi, T., and D. A. Randall, 2012: Cooling of entrained parcels in a large-eddy simulation. J. Atmos. Sci., 69, 11181136. 